\title{
A ROE-TYPE SCHEME FOR TWO-PHASE SHALLOW GRANULAR FLOWS OVER VARIABLE TOPOGRAPHY
}

\author{
Marica Pelanti $^{1}$, François Bouchut $^{2}$ and Anne Mangeney ${ }^{3,4}$
}

\begin{abstract}
We study a depth-averaged model of gravity-driven flows made of solid grains and fluid, moving over variable basal surface. In particular, we are interested in applications to geophysical flows such as avalanches and debris flows, which typically contain both solid material and interstitial fluid. The model system consists of mass and momentum balance equations for the solid and fluid components, coupled together by both conservative and non-conservative terms involving the derivatives of the unknowns, and by interphase drag source terms. The system is hyperbolic at least when the difference between solid and fluid velocities is sufficiently small. We solve numerically the one-dimensional model equations by a high-resolution finite volume scheme based on a Roe-type Riemann solver. Wellbalancing of topography source terms is obtained via a technique that includes these contributions into the wave structure of the Riemann solution. We present and discuss several numerical experiments, including problems of perturbed steady flows over non-flat bottom surface that show the efficient modeling of disturbances of equilibrium conditions.
\end{abstract}

Mathematics Subject Classification. 65M99, 76T25.

Received November 9, 2007.

Published online July 30, 2008.

\section{INTRODUCTION}

In this paper we study a depth-averaged two-phase flow model for gravity-driven mixtures of solid material and fluid. Our main interest, which was the original motivation for the present work, is the application to gravitational geophysical flows such as avalanches and debris flows. These flows are granular masses that often contain both solid components and a variable amount of interstitial fluid.

Following the pioneering work of Savage and Hutter [45,46], in recent years great advances have been made in the mathematical and numerical modeling of granular geophysical flows by means of depth-averaged or thin layer models, which are based on the small aspect ratio of typical flows (small characteristic flow depth $H$ compared to the characteristic flow length $L$ ). Savage and Hutter [45] began studying the one-dimensional

\footnotetext{
Keywords and phrases. Granular flows, two-phase flows, thin layer approximation, non-conservative systems, numerical model, finite volume schemes, Riemann solvers, well-balanced schemes.

1 Département de Mathématiques et Applications, École Normale Supérieure, 45 rue d'Ulm, 75230 Paris Cedex 05, France. Marica.Pelanti@ens.fr

2 CNRS and Département de Mathématiques et Applications, École Normale Supérieure, 45 rue d'Ulm, 75230 Paris Cedex 05, France. Francois.Bouchut@ens.fr

3 Équipe de Sismologie, Institut de Physique du Globe de Paris, 4 place Jussieu, 75252 Paris Cedex 05, France.

mangeney@ipgp.jussieu.fr

${ }^{4}$ Institute for Nonlinear Science, University of California San Diego, 9500 Gilman Drive, La Jolla, CA 92093-0402, USA.
} 
motion of dry granular material down inclines. Later their approach has been developed further by numerous authors, with extensions to two dimensions [18] and generalizations to flows on more complex bottom topography $[5,9,15,20,35,40,41]$. Numerical algorithms implementing these depth-averaged models have been able to reproduce many experimental tests and a large range of geological observations with significant success $[8,21,29-31,35,39,41,49,53]$.

Despite the important progress done, one open problem that still needs to be addressed in the description of the debris flow mechanics is an accurate modeling of pore fluid effects. Interaction forces between solid grains and interstitial fluid influence the rheological behavior of the moving mass and may play an important role in deformation processes, flow mobility and run-out [19,22].

Considerable part of the literature on granular flow modeling is limited to single-phase dry granular masses, from early studies $[15,45,46]$ to more recent works [30,35,40,42]. Iverson [19] and Iverson and Denlinger [20] made an effort to take into account intergranular fluid effects in the flowing material, and they developed a solid-fluid mixture theory based on the simplifying assumptions of constant porosity (fluid volume fraction) and equality of fluid velocity to solid velocity. Here the flow is described by a set of balance equations for the mass and for the momentum of the mixture, which formally appears as a single-phase model with a stress term accounting for contributions from the two constituents. This mixture formulation lacks an inherent description of the pore fluid motion and the model needs to be supplemented with some specification of the pore fluid pressure evolution. A pore fluid pressure advection-diffusion equation was assumed in [20], based on experimental measurements. Numerical applications of this model to laboratory avalanches and debris flume experiments are reported in [8]. Among later works in the context of solid-fluid mixture theory, let us also mention the more recent paper [41] of Pudasaini and co-workers, who propose a model for debris flows down general channels by using the same simplifying hypotheses of constant mixture density and equality of phase velocities as Iverson and Denlinger.

Making a significant step forward with respect to mixture models, Pitman and Le [38] have recently presented a novel depth-averaged two-phase model for debris flows and avalanches that contains mass and momentum equations for both the solid and fluid component. This implicitly provides equations for the velocities of both phases and for porosity. However, in this work the authors propose a numerical method only for a reduced system that ignores fluid inertial terms.

We present here a mathematical and numerical two-phase granular flow model over variable topography that follows the approach of Pitman and Le [38]. In the present paper we limit our study to the one-dimensional case. We start recalling the work of Pitman and Le in Section 2, and in Section 3 we introduce our mathematical model. As in [38], our system of equations expresses mass and momentum balances for both the solid and fluid phase, and it includes interphase drag terms. However, the formulation of our two-phase system differs from the original one in [38] in the description of the fluid phase dynamics, and it has the new property of recovering a conservative equation for the momentum of the mixture. In Section 4 we analyze the eigenstructure of our model and we derive sufficient conditions for hyperbolicity. In particular, we show that the model system is hyperbolic at least for flow regimes characterized by sufficiently small phase velocity differences. Based on this, for the system solution we will assume solid and fluid velocity values enough close for the equations to be hyperbolic, a reasonable hypothesis for the applications to debris flows we are interested in, e.g. [19].

In Section 5 we briefly present a reduced model, derived by assuming that drag forces are strong enough to drive instantaneously solid and fluid velocities to equilibrium. The only interest for this reduced system is that it allows a much simpler mathematical analysis than the full two-phase system and an easy derivation of exact solutions. This reduced model will be uniquely used for comparison with our two-phase model in the numerical tests.

We then illustrate the numerical scheme for the solution of our system in Sections 6-9. We employ a finite volume method belonging to the family of upwind schemes based on Riemann solvers (Godunov-type methods) $[12,26,50]$. Such a class of methods has been applied with considerable success for granular flow computations with single-phase and mixture models [8,21,29,35,53].

A specific numerical difficulty for our two-phase system comes from the presence of non-conservative terms involving the derivatives of the unknowns that couple together the sets of equations of the solid and fluid phase. 
This will be addressed in Section 6, where we describe the Roe-type solver that we employ to approximate local Riemann problems. Section 7 then explains how we formulate our scheme into the framework of the high-resolution wave-propagation algorithms of [24].

Another numerical difficulty in the approximation of our system is to ensure well-balancing of bottom topography terms, in order for the scheme to maintain steady state conditions at the discrete level, and to model correctly small perturbations from steady states. Preserving equilibrium states and resolving accurately deviations from them is a well known numerical problem that in general arises in the approximation of systems with sources that at steady states balance some spatial gradients of the unknowns. Schemes for such systems that approximate correctly steady and nearly steady conditions are called well-balanced, and there exists a very extensive literature on the subject, see for instance [2,3,13,16,25,33,34,52] and the monograph [4]. Our treatment of bottom surface terms is explained in Section 8, while Section 9 describes the modeling of interphase drag forces. We finally report results of several numerical tests in Section 10, and we conclude the paper by writing some remarks.

\section{The Pitman-Le two-Phase Model}

We briefly recall in this section the modeling approach of Pitman and Le [38], referring to the original paper for details. Pitman and Le consider a thin layer of a mixture of solid granular material and interstitial fluid moving over a smooth basal surface. Solid and fluid components are assumed incompressible, with constant specific densities $\rho_{s}$ and $\rho_{f}<\rho_{s}$, respectively. Mass and momentum equations for the two constituents are written based on the two-phase model of Anderson and Jackson [1]:

$$
\begin{aligned}
& \partial_{t}\left(\rho_{s} \varphi\right)+\nabla \cdot\left(\rho_{s} \varphi \mathcal{V}_{s}\right)=0 \\
& \rho_{s} \varphi\left(\partial_{t} \mathcal{V}_{s}+\left(\mathcal{V}_{s} \cdot \nabla\right) \mathcal{V}_{s}\right)=\nabla \cdot T_{s}+\varphi \nabla \cdot T_{f}+\mathcal{I}+\rho_{s} \varphi \mathfrak{g} \\
& \partial_{t}\left(\rho_{f}(1-\varphi)\right)+\nabla \cdot\left(\rho_{f}(1-\varphi) \mathcal{V}_{f}\right)=0 \\
& \rho_{f}(1-\varphi)\left(\partial_{t} \mathcal{V}_{f}+\left(\mathcal{V}_{f} \cdot \nabla\right) \mathcal{V}_{f}\right)=(1-\varphi) \nabla \cdot T_{f}-\mathcal{I}+\rho_{f}(1-\varphi) \mathfrak{g}
\end{aligned}
$$

Above $\varphi$ is the solid volume fraction, $\mathcal{V}_{s}$ and $\mathcal{V}_{f}$ are the solid and fluid vectorial velocities, $T_{s}$ and $T_{f}$ the solid and fluid stress tensors. Note that here we use the convention of denoting compressive stresses as negative, differently from [38]. Furthermore, $\mathfrak{g}$ is the gravity acceleration vector, and $\mathcal{I}$ represents all the non-buoyancy interaction forces. Interphase drag is assumed to be the only contribution to $\mathcal{I}$, and a standard form of the drag force is used, $\mathcal{I}=\mathcal{D}\left(\mathcal{V}_{f}-\mathcal{V}_{s}\right)$, where $\mathcal{D}$ is a drag function. Typically $[32,51], \mathcal{D}=\mathcal{D}\left(\varphi,\left|\mathcal{V}_{f}-\mathcal{V}_{s}\right| ; \sigma\right)$, where $\sigma$ is a vector of physical parameters such as the specific densities $\rho_{s}$ and $\rho_{f}$, the fluid dynamic viscosity and the solid particle diameter. In particular, Pitman and Le take $\mathcal{D}=|\mathfrak{g}|(1-\varphi)^{1-\Sigma} \varphi\left(\rho_{s}-\rho_{f}\right) / v_{T}$, where $v_{T}$ is a characteristic velocity, and $\Sigma$ depends on the Reynolds number of the flow.

In order to complete the formulation, a specification of material stresses is needed. The fluid is assumed inviscid, and the only fluid stress considered is a pressure. The solid, as in the Savage-Hutter theory, is modeled as a Mohr-Coulomb material. A Coulomb friction law is used, expressing collinearity of shear stresses and normal stresses through a friction coefficient $\nu=\tan \delta^{f}$, where $\delta^{\mathrm{f}}$ is the local angle of friction. An earth pressure relation is then assumed for solid lateral normal stresses. Here we will describe only the one-dimensional thin layer model, with motion in a direction $x$. In this case, denoting with $z$ the normal direction, the relations for the relevant solid stress components are $T_{s}^{x z}= \pm \nu T_{s}^{z z}$ (Coulomb friction law), with the sign taken so that the shear traction opposes the solid sliding motion, and $T_{s}^{x x}=K T_{s}^{z z}$ (earth pressure relation), where $K$ is the so-called earth pressure coefficient [43]. For simplicity, here we will set $K=1$. The free upper surface of the flow is stress-free for both constituents. As for kinematic boundary conditions, material surface conditions at the upper free surface, and tangent motion conditions at the base are imposed for each phase.

Making use of the shallow flow assumption $H / L \ll 1(H=$ characteristic thickness, $L=$ characteristic flow length), the governing equations (2.1) are then suitably scaled and averaged over flow depth. We report below the resulting model equations, omitting the lengthy procedure of their derivation ( $c f$. [38]). Here we will make 
the further assumption of small topography slope, so that the direction tangent to the bottom surface can be approximated by the horizontal direction. Then, the depth-averaged two-phase model of Pitman and Le can be written in the form:

$$
\begin{aligned}
& \partial_{t}(\bar{\varphi} h)+\partial_{x}\left(\bar{\varphi} h \bar{v}_{s}\right)=0 \\
& \partial_{t}\left(\bar{\varphi} h \bar{v}_{s}\right)+\partial_{x}\left(\bar{\varphi} h \bar{v}_{s}^{2}+g \frac{1-\gamma}{2} \bar{\varphi} h^{2}\right)+\gamma \bar{\varphi} \frac{g}{2} \partial_{x} h^{2}=-g \bar{\varphi} h \partial_{x} b-\operatorname{sgn}\left(\bar{v}_{s}\right) \nu^{\mathrm{b}} g(1-\gamma) \bar{\varphi} h+\gamma D h\left(\bar{v}_{f}-\bar{v}_{s}\right) \\
& \partial_{t} h+\partial_{x}\left(\bar{\varphi} h \bar{v}_{s}+(1-\bar{\varphi}) h \bar{v}_{f}\right)=0 \\
& \partial_{t}\left(h \bar{v}_{f}\right)+\partial_{x}\left(h \bar{v}_{f}^{2}\right)+\frac{g}{2} \partial_{x} h^{2}=-g h \partial_{x} b-\frac{D}{1-\bar{\varphi}} h\left(\bar{v}_{f}-\bar{v}_{s}\right)
\end{aligned}
$$

Above $h$ is the flow depth, $\bar{\varphi}$ the depth-averaged solid volume fraction, $\bar{v}_{s}$ and $\bar{v}_{f}$ are the solid and fluid averaged velocities, respectively, $g=|\mathfrak{g}|$ (gravity constant), and, moreover, we have introduced the ratio

$$
\gamma=\frac{\rho_{f}}{\rho_{s}}<1
$$

Furthermore, $b(x)$ describes the bottom topography, $\nu^{\mathrm{b}}=\tan \delta^{\text {bed }}$, where $\delta^{\text {bed }}$ is the basal friction angle, and finally $D=\overline{\mathcal{D}} / \rho_{f}$, where $\overline{\mathcal{D}}$ is an average of $\mathcal{D}\left(\varphi,\left|\mathcal{V}_{f}-\mathcal{V}_{s}\right| ; \sigma\right)$. As suggested in [38], it is reasonable to adopt the same form of $\mathcal{D}$ as a drag relation for the average quantities, that is we take $D=\frac{1}{\rho_{f}} \mathcal{D}\left(\bar{\varphi},\left|\bar{v}_{f}-\bar{v}_{s}\right| ; \sigma\right)$. For the specific choice of $\mathcal{D}$ in [38], this gives $D=g(1-\bar{\varphi})^{1-\Sigma} \bar{\varphi}(1 / \gamma-1) / v_{T}$.

Note that equations (2.2a) and (2.2c) express mass conservation for the solid phase and for the mixture, respectively, while equations $(2.2 \mathrm{~b})$ and $(2.2 \mathrm{~d})$ express solid and fluid momentum balances.

For the two-phase model above simple explicit expressions of the eigenvalues are not available, and approximate formulas are derived in [38] by using a perturbation expansion in $\sqrt{H / L}$, starting from the model written in normalized form. The authors claim hyperbolicity of the model equations at least for $H / L$ sufficiently small.

\section{A NEW MODEL FORMULATION}

The two-phase shallow flow model that we present in this section is a variant of the Pitman-Le model, and it differs from the original work of [38] in the description of the fluid and mixture momentum balance. Here we prefer a symmetric formulation of the solid and fluid depth-averaged equations, and we express mass conservation and momentum balance for each constituent by writing equations for the mass variables $\bar{\varphi} h$ and $(1-\bar{\varphi}) h$, and for the momenta $\bar{\varphi} h \bar{v}_{s}$ and $(1-\bar{\varphi}) h \bar{v}_{f}$. Most significantly, we integrate the fluid momentum equation in a different form, to derive the equation for $(1-\bar{\varphi}) h \bar{v}_{f}$ that will replace $(2.2 \mathrm{~d})$. Note that the motion equations $(2.1 \mathrm{~b})$ and $(2.1 \mathrm{~d})$ for $\mathcal{V}_{s}$ and $\mathcal{V}_{f}$ can be reformulated as equations for $\varphi \mathcal{V}_{s}$ and $(1-\varphi) \mathcal{V}_{f}$, respectively, by rewriting the left-hand side in the equivalent conservative form $\rho_{\theta}\left(\partial_{t}\left(\varphi_{\theta} \mathcal{V}_{\theta}\right)+\nabla \cdot\left(\varphi_{\theta} \mathcal{V}_{\theta} \otimes \mathcal{V}_{\theta}\right)\right), \theta=s, f$, $\varphi_{s}=\varphi, \varphi_{f}=(1-\varphi)$. To express the $x$-momentum balances, Pitman and Le integrate a solid motion equation for $\varphi \mathcal{V}_{s}^{(x)}$, and a fluid motion equation for $\mathcal{V}_{f}^{(x)}$, where $\mathcal{V}_{\theta}^{(x)}$ is the $x$-component of $\mathcal{V}_{\theta}, \theta=s, f$. Instead, for the fluid phase we average an equation for $(1-\varphi) \mathcal{V}_{f}^{(x)}$, thus employing symmetric terms for the two phases. Although the two approaches use equivalent forms of (2.1d), they lead to slightly different expressions of the fluid momentum balance due to the averaging approximations. More precisely, in the integration process the average of any product of variables is approximated as the product of the corresponding depth-averaged variables. See discussion in [38] for an estimation of errors in averaging. 
Omitting hereafter overbars to simplify the notation, our model takes the form:

$$
\begin{aligned}
& \partial_{t}(\varphi h)+\partial_{x}\left(\varphi h v_{s}\right)=0 \\
& \partial_{t}\left(\varphi h v_{s}\right)+\partial_{x}\left(\varphi h v_{s}^{2}+g \frac{1-\gamma}{2} \varphi h^{2}\right)+\gamma \varphi \frac{g}{2} \partial_{x} h^{2}=-g \varphi h \partial_{x} b-\operatorname{sgn}\left(v_{s}\right) \nu^{\mathrm{b}} g(1-\gamma) \varphi h+\gamma D h\left(v_{f}-v_{s}\right) \\
& \partial_{t}((1-\varphi) h)+\partial_{x}\left((1-\varphi) h v_{f}\right)=0 \\
& \partial_{t}\left((1-\varphi) h v_{f}\right)+\partial_{x}\left((1-\varphi) h v_{f}^{2}\right)+(1-\varphi) \frac{g}{2} \partial_{x} h^{2}=-g(1-\varphi) h \partial_{x} b-D h\left(v_{f}-v_{s}\right)
\end{aligned}
$$

with $D=\frac{1}{\rho_{f}} \mathcal{D}\left(\varphi,\left|v_{f}-v_{s}\right| ; \sigma\right)$. The mass equations (3.1a) and (3.1c) are equivalent to the couple (2.2a) and (2.2c). This in particular implies that the solid volume fraction is governed by an equation of the same form in the two models:

$$
\partial_{t} \varphi+\varphi(1-\varphi)\left(v_{s}-v_{f}\right) \frac{1}{h} \partial_{x} h+\left((1-\varphi) v_{s}+\varphi v_{f}\right) \partial_{x} \varphi+\varphi(1-\varphi) \partial_{x}\left(v_{s}-v_{f}\right)=0
$$

While the solid momentum balance is also expressed by the same equation, the dynamics of the fluid phase is described in a different way. By considering that

$$
\partial_{t}\left((1-\varphi) h v_{f}\right)+\partial_{x}\left((1-\varphi) h v_{f}^{2}\right)=(1-\varphi)\left(\partial_{t}\left(h v_{f}\right)+\partial_{x}\left(h v_{f}^{2}\right)\right)+h v_{f}\left(\partial_{t}(1-\varphi)+v_{f} \partial_{x}(1-\varphi)\right)
$$

we see that the fluid momentum equation $(2.2 \mathrm{~d})$ is equivalent to the equation $(3.1 \mathrm{~d})$ with the addition of a term $\tau \equiv-h v_{f}\left(\partial_{t}(1-\varphi)+v_{f} \partial_{x}(1-\varphi)\right)$ on the left-hand side. By using mass conservation, this term can be also written as $\tau=-(1-\varphi) v_{f} \partial_{x}\left(\varphi h\left(v_{s}-v_{f}\right)\right)$. Note that the two models are coincident if the volume fraction $\varphi$ is constant in space and time, or if we assume instantaneous velocity equilibrium between the two constituents $\left(v_{s}=v_{f}\right)$. In particular, both models reduce to classical single-phase shallow water equations for constant $\varphi$ and $v_{s}=v_{f}$.

The different expression of fluid momentum balance entails a different description of the mixture dynamics. Consistently with the equations before averaging (2.1), and with the expected physical behavior, our model has the property of recovering a conservative equation for the momentum of the mixture, which has the form

$$
\begin{aligned}
\partial_{t}\left(\left(\varphi v_{s}+\gamma(1-\varphi) v_{f}\right) h\right)+\partial_{x}\left(\left(\varphi v_{s}^{2}+\gamma(1-\varphi) v_{f}^{2}\right) h+\right. & \left.\frac{g}{2}(\varphi+\gamma(1-\varphi)) h^{2}\right)= \\
& -g(\varphi+\gamma(1-\varphi)) h \partial_{x} b-\operatorname{sgn}\left(v_{s}\right) \nu^{\mathrm{b}} g(1-\gamma) \varphi h
\end{aligned}
$$

From the Pitman-Le equations (2.2) we derive a mixture momentum equation of the form above, with an additional non-conservative term $\gamma \tau$ on the left-hand side. Therefore, it doesn't seem possible to recover a mixture momentum equation in conservation form for the model in [38].

\subsection{Formulation in $\boldsymbol{h}_{\boldsymbol{s}}, \boldsymbol{h}_{\boldsymbol{f}}$}

We now rewrite our model by expressing quantities containing the variables $\varphi$ and $h$ in terms of the conserved quantities $h_{s} \equiv \varphi h$ and $h_{f} \equiv(1-\varphi) h$. We neglect friction, which will not be considered hereafter. Manipulating suitably the equations $(3.1)$, and setting $q=\left(h_{s}, h_{s} v_{s}, h_{f}, h_{f} v_{f}\right)^{\mathrm{T}}$, we obtain the system

$$
\partial_{t} q+\partial_{x} f(q)+w\left(q, \partial_{x} q\right)=\psi^{\mathrm{b}}(q)+\psi^{\mathrm{D}}(q)
$$


where

$$
f(q)=\left(\begin{array}{c}
h_{s} v_{s} \\
h_{s} v_{s}^{2}+\frac{g}{2} h_{s}^{2}+g \frac{1-\gamma}{2} h_{s} h_{f} \\
h_{f} v_{f} \\
h_{f} v_{f}^{2}+\frac{g}{2} h_{f}^{2}
\end{array}\right), \quad w\left(q, \partial_{x} q\right)=\left(\begin{array}{c}
0 \\
\gamma g h_{s} \partial_{x} h_{f} \\
0 \\
g h_{f} \partial_{x} h_{s}
\end{array}\right)
$$

and the topography and drag source terms are given by

$$
\psi^{\mathrm{b}}(q)=-\left(\begin{array}{c}
0 \\
g h_{s} \partial_{x} b \\
0 \\
g h_{f} \partial_{x} b
\end{array}\right), \quad \psi^{\mathrm{D}}(q)=\left(\begin{array}{c}
0 \\
\gamma F^{\mathrm{D}} \\
0 \\
-F^{\mathrm{D}}
\end{array}\right), \quad F^{\mathrm{D}}=D\left(h_{s}+h_{f}\right)\left(v_{f}-v_{s}\right)
$$

Above, we have put into evidence the conservative portion of the system $\partial_{x} f(q)$, and the non-conservative term $w\left(q, \partial_{x} q\right)$. An interesting feature of this formulation in terms of $h_{s}, h_{f}$ is that (with $D \equiv 0$ ) it has a formal mathematical similarity with the classical two-layer shallow water model system (e.g. $[6,7,34,54])$, if we interpret $h_{s}$ and $h_{f}$ as the depths of the two flow layers and $v_{s}$ and $v_{f}$ as the corresponding velocities. The only difference with the two-layer system is the additional conservative cross term $\partial_{x}\left(g \frac{1-\gamma}{2} h_{s} h_{f}\right)$ in the solid momentum equation of our two-phase model.

Let us also write system (3.5) in quasi-linear form for later reference:

$$
\partial_{t} q+A(q) \partial_{x} q=\psi^{\mathrm{b}}(q)+\psi^{\mathrm{D}}(q)
$$

where

$$
A(q)=\left(\begin{array}{cccc}
0 & 1 & 0 & 0 \\
-v_{s}^{2}+g h_{s}+g \frac{1-\gamma}{2} h_{f} & 2 v_{s} & g \frac{1+\gamma}{2} h_{s} & 0 \\
0 & 0 & 0 & 1 \\
g h_{f} & 0 & -v_{f}^{2}+g h_{f} & 2 v_{f}
\end{array}\right)
$$

Finally, the equation for the mixture momentum $m=h_{s} v_{s}+\gamma h_{f} v_{f}$ can be written as

$$
\partial_{t} m+\partial_{x} f_{\mathrm{m}}(q)=-g\left(h_{s}+\gamma h_{f}\right) \partial_{x} b,
$$

where

$$
f_{\mathrm{m}}(q)=f^{(2)}(q)+\gamma f^{(4)}(q)+\gamma g h_{s} h_{f}=h_{s} v_{s}^{2}+\gamma h_{f} v_{f}^{2}+\frac{g}{2}\left(h_{s}^{2}+\gamma h_{f}^{2}\right)+g \frac{1+\gamma}{2} h_{s} h_{f} .
$$




\subsection{Steady states}

Steady state conditions for model (3.5) are

$$
\begin{aligned}
& h_{s} v_{s}=\text { const., } \\
& h_{f} v_{f}=\text { const., } \\
& \frac{v_{f}^{2}}{2}+g\left(h_{s}+h_{f}+b\right)=\text { const., } \\
& h_{s} \partial_{x}\left(\frac{v_{f}^{2}}{2}-\frac{v_{s}^{2}}{2}+g \frac{1-\gamma}{2} h_{f}\right)-h_{f} \partial_{x}\left(g \frac{1-\gamma}{2} h_{s}\right)=0 .
\end{aligned}
$$

In the particular case of steady state at rest $\left(v_{s}=v_{f}=0\right)$ these conditions simplify to $h_{s}+h_{f}+b=$ const. and $h_{s} / h_{f}=$ const. In terms of the significant physical variables $h=h_{s}+h_{f}$ and $\varphi=h_{s} /\left(h_{s}+h_{f}\right)$, this means that equilibrium at rest is characterized by

$$
h+b=\text { const. } \quad \text { and } \quad \varphi=\text { const. }
$$

\section{Eigenstructure AND hyperbolicity}

\subsection{Eigenvalue analysis}

We study here the eigenvalues of our two-phase model, assuming $h>0$. Hence, we look for the roots of the characteristic polynomial $P(\lambda)=\operatorname{det}(A-\lambda I)$ of the matrix $A$ of system (3.6). For the purpose of this analysis, it is convenient to use the variables $h, \varphi$, and to introduce the quantities

$$
a=\sqrt{g h} \quad \text { and } \quad \beta=\sqrt{\frac{1}{2}(1-\varphi)(1-\gamma)}<1
$$

The polynomial $P(\lambda)$ has the quartic form $P(\lambda)=\sum_{k=0}^{4} b_{k} \lambda^{k}$, where the coefficients $b_{k}$ can be expressed as

$$
\begin{aligned}
& b_{4}=1, \\
& b_{3}=-2\left(v_{s}+v_{f}\right), \\
& b_{2}=\left(v_{s}+v_{f}\right)^{2}+2 v_{s} v_{f}-a^{2}\left(1+\beta^{2}\right), \\
& b_{1}=-2 v_{s} v_{f}\left(v_{s}+v_{f}\right)+2 a^{2}\left(v_{s}+\beta^{2} v_{f}\right)-2 a^{2} \varphi\left(v_{s}-v_{f}\right), \\
& b_{0}=v_{s}^{2} v_{f}^{2}-a^{2}\left(v_{s}^{2}+\beta^{2} v_{f}^{2}\right)+a^{4} \beta^{2}+a^{2} \varphi\left(v_{s}^{2}-v_{f}^{2}\right) .
\end{aligned}
$$

In general, as for the original Pitman-Le model, simple explicit expressions of the roots $\lambda_{k}$ of $P(\lambda), k=1, \ldots, 4$, cannot be derived. In the particular case of equality of solid and fluid velocities, $v_{f}=v_{s} \equiv v$, the four roots of $P(\lambda)$ are real and distinct $(\varphi \neq 1)$, and given by

$$
\lambda_{1,4}=v \mp a \quad \text { and } \quad \lambda_{2,3}=v \mp a \beta .
$$

Other particular cases are:

- $\varphi=0$, for which the eigenvalues are $v_{f} \mp a, v_{s} \mp a \beta$, with $\beta=\sqrt{\frac{1-\gamma}{2}}$;

- $\varphi=1$, for which we find the two distinct eigenvalues $v_{s} \mp a$ and the double eigenvalue $v_{f}$. As a special sub-case, let us remark that if $\left|v_{s}-v_{f}\right|=a$ then there are only two distinct eigenvalues.

Although if $\varphi=0$ (or $\varphi=1$ ) everywhere in the domain then the velocity $v_{s}$ (resp. $v_{f}$ ) is not defined, note that the eigenvalues expressions just above for $\varphi=0$ and $\varphi=1$ are still meaningful whenever $v_{s}$ and $v_{f}$ have a determined value (e.g. at the edges of rarefactions where $\varphi$ or $(1-\varphi)$ may vanish). 
Let us now analyze the general case. We can state the following result:

Proposition 4.1. Matrix $A$ has always at least two real eigenvalues $\lambda_{1,4}$, and moreover, the eigenvalues $\lambda_{k}$ of $A, k=1, \ldots, 4$, satisfy the following inequalities:

$$
v_{\min }-a \leq \lambda_{1} \leq \mathfrak{R e}\left(\lambda_{2}\right) \leq \mathfrak{R e}\left(\lambda_{3}\right) \leq \lambda_{4} \leq v_{\max }+a,
$$

where $v_{\min } \equiv \min \left(v_{f}, v_{s}\right), v_{\max } \equiv \max \left(v_{f}, v_{s}\right)$, and $\mathfrak{R e}(\cdot)$ denotes the real part. Furthermore:

(i) If $\left|v_{s}-v_{f}\right| \leq 2 a \beta$ or $\left|v_{s}-v_{f}\right| \geq 2 a$ then all the eigenvalues are real. If these inequalities are strictly satisfied, and if $\varphi \neq 1$, then the eigenvalues are also distinct, and system (3.6) is strictly hyperbolic.

(ii) If $2 a \beta<\left|v_{s}-v_{f}\right|<2 a$ then the internal eigenvalues $\lambda_{2,3}$ may be complex.

Proof. To study the roots of $P(\lambda)$ it is useful to introduce the quartic polynomials $p^{s}(\lambda), p^{f}(\lambda)$ that have roots $v_{f} \pm a, v_{s} \pm a \beta$, and $v_{s} \pm a, v_{f} \pm a \beta$, respectively:

$$
\begin{aligned}
& p^{f}(\lambda)=\left(\lambda-\left(v_{f}-a\right)\right)\left(\lambda-\left(v_{f}+a\right)\right)\left(\lambda-\left(v_{s}-a \beta\right)\right)\left(\lambda-\left(v_{s}+a \beta\right)\right), \\
& p^{s}(\lambda)=\left(\lambda-\left(v_{s}-a\right)\right)\left(\lambda-\left(v_{s}+a\right)\right)\left(\lambda-\left(v_{f}-a \beta\right)\right)\left(\lambda-\left(v_{f}+a \beta\right)\right) .
\end{aligned}
$$

These functions both intersect $P(\lambda)$ at $\bar{v}=\frac{v_{s}+v_{f}}{2}$, and, moreover, they differ from $P(\lambda)$ only for terms up to first order in $\lambda$ :

$$
\begin{aligned}
& P(\lambda)-p^{f}(\lambda)=-2 a^{2} \varphi\left(v_{s}-v_{f}\right)\left(\lambda-\frac{v_{s}+v_{f}}{2}\right) \\
& P(\lambda)-p^{s}(\lambda)=a^{2}(1-\varphi)(1+\gamma)\left(v_{s}-v_{f}\right)\left(\lambda-\frac{v_{s}+v_{f}}{2}\right) .
\end{aligned}
$$

The common coordinates of the inflection points of $P(\lambda), p^{s}(\lambda)$ and $p^{f}(\lambda)$ are given by:

$$
\omega^{1,2}=\bar{v} \mp \frac{1}{2} \sqrt{\frac{1}{3}\left(v_{s}-v_{f}\right)^{2}+\frac{2}{3} a^{2}\left(1+\beta^{2}\right)},
$$

and the curves are convex for $\lambda \in\left(-\infty, \omega^{1}\right) \cup\left(\omega^{2},+\infty\right)$, and concave for $\lambda \in\left(\omega^{1}, \omega^{2}\right)$. Note that if $v_{s}=v_{f}$ then $p^{f} \equiv p^{s} \equiv P$. Moreover, $P \equiv p^{f}$ for $\varphi=0$, and $P \equiv p^{s}$ for $\varphi=1$. Then it is clear that in these special cases the eigenvalues have the expressions anticipated earlier in this section (see (4.3) and following relations), and that the statements of Proposition 4.1 are satisfied.

Let us assume now $v_{s} \neq v_{f}, \varphi \neq 0, \varphi \neq 1$. Then $\bar{v}$ is the only point of intersection of the polynomials. Moreover:

$$
\begin{aligned}
& p^{f}(\lambda)<P(\lambda)<p^{s}(\lambda) \quad \text { if } v_{s}>v_{f} \text { and } \lambda<\bar{v} \quad \text { or } \quad \text { if } v_{s}<v_{f} \text { and } \lambda>\bar{v} \text {, } \\
& p^{s}(\lambda)<P(\lambda)<p^{f}(\lambda) \quad \text { if } v_{s}>v_{f} \text { and } \lambda>\bar{v} \quad \text { or } \quad \text { if } v_{s}<v_{f} \text { and } \lambda<\bar{v} \text {, }
\end{aligned}
$$

which means that the polynomial $P(\lambda)$ is bounded by $p^{s}(\lambda)$ and $p^{f}(\lambda)$. As a consequence, all the real roots of $P(\lambda)$ will be bounded by $v_{\min }-a$ from below, and by $v_{\max }+a$ from above. See Figure 1 . We also deduce that there are at least two real roots,

$$
\lambda_{1} \in\left(v_{\min }-a, \min \left(v_{\max }-a, v_{\min }-a \beta\right)\right) \quad \text { and } \quad \lambda_{4} \in\left(\max \left(v_{\min }+a, v_{\max }+a \beta\right), v_{\max }+a\right) .
$$

Moreover, if the other two roots $\lambda_{2,3}$ are also real, then they will be located in the interval:

$$
\lambda_{2,3} \in\left(\min \left(v_{\max }-a, v_{\min }-a \beta\right), \max \left(v_{\min }+a, v_{\max }+a \beta\right)\right) .
$$




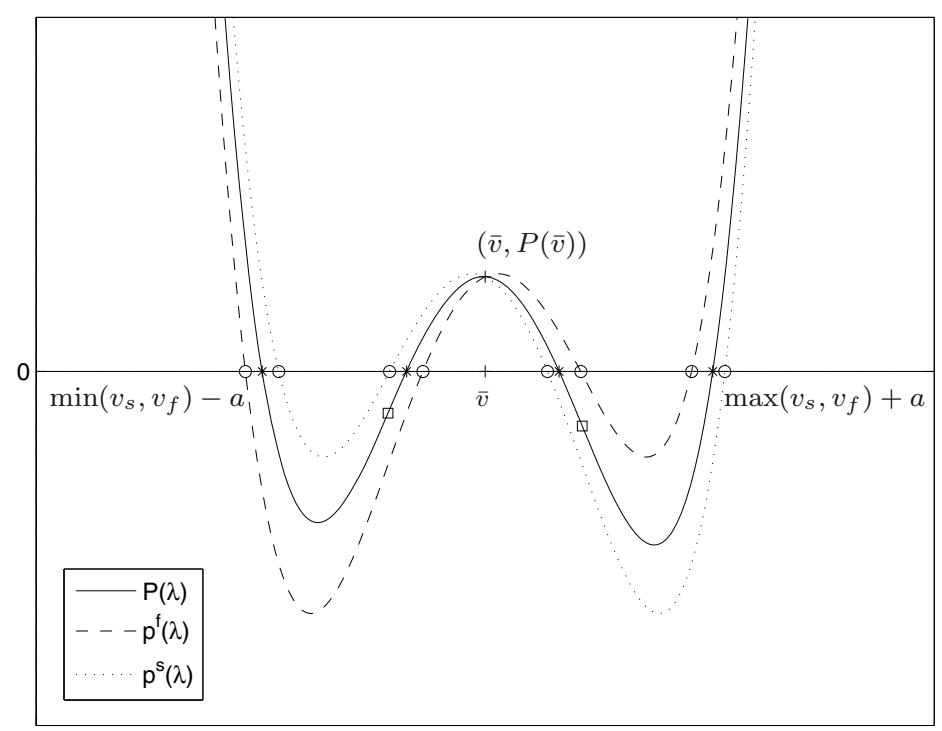

Figure 1. Characteristic polynomial $P(\lambda)$ and curves $p^{s}(\lambda), p^{f}(\lambda)$ for a case in which the roots of $P(\lambda)$ are real. Stars $(*)$ indicate the roots of $P(\lambda)$, circles $(0)$ the roots of $p^{s}(\lambda)$ and $p^{f}(\lambda)$, and squares $(\square)$ the inflection points $\left(\omega^{i}, P\left(\omega^{i}\right)\right), i=1,2$.

Therefore, we have in this case $\lambda_{1}<\lambda_{2} \leq \lambda_{3}<\lambda_{4}$. Let us assume now that $\lambda_{2,3}$ are complex. In general, the following relation between the roots of $P(\lambda)$ and the coefficient $b_{3}$ holds:

$$
\sum_{k=1}^{4} \lambda_{k}=-b_{3}=2\left(v_{s}+v_{f}\right)
$$

In the case under consideration we can write

$$
\mathfrak{R e}\left(\lambda_{2}\right)=\mathfrak{R e}\left(\lambda_{3}\right)=v_{s}+v_{f}-\frac{1}{2}\left(\lambda_{1}+\lambda_{4}\right) .
$$

Since from (4.9) we have $v_{\min }<\frac{1}{2}\left(\lambda_{1}+\lambda_{4}\right)<v_{\max }$ we then obtain by (4.12)

$$
v_{\min }<\mathfrak{R e}\left(\lambda_{2}\right)=\mathfrak{R e}\left(\lambda_{3}\right)<v_{\max } .
$$

Hence, again through (4.9), we deduce

$$
\lambda_{1}<\mathfrak{R e}\left(\lambda_{2}\right)=\mathfrak{R e}\left(\lambda_{3}\right)<\lambda_{4}
$$

This completes the proof of relations (4.4). Note that for $\varphi \neq 1$ we have the more strict inequalities $\lambda_{1}<$ $\mathfrak{R e}\left(\lambda_{2}\right) \leq \mathfrak{R e}\left(\lambda_{2}\right)<\lambda_{4}$

Let us now investigate the conditions for real eigenvalues. From the behavior of $P(\lambda)$ we observe that a necessary and sufficient condition for having four real roots is the positivity of the local maximum in the region of concavity. Unfortunately, no simple expression can be obtained to characterize this point. However, noticing that $\omega^{1}<\bar{v}<\omega^{2}$, we can easily obtain sufficient conditions by imposing positivity of $P(\bar{v})$ :

$$
P(\bar{v})=p^{f}(\bar{v})=p^{s}(\bar{v})=\left[\left(\frac{v_{s}-v_{f}}{2}\right)^{2}-a^{2}\right]\left[\left(\frac{v_{s}-v_{f}}{2}\right)^{2}-a^{2} \beta^{2}\right] \geq 0 .
$$


This gives

$$
\left|v_{s}-v_{f}\right| \leq 2 a \beta, \quad \text { or } \quad\left|v_{s}-v_{f}\right| \geq 2 a .
$$

With strict inequalities these conditions ensure also that $\lambda_{2}<\lambda_{3}$, so that, for $\varphi \neq 1$, the eigenvalues will be all distinct. Then, system (3.6) will be strictly hyperbolic.

To show an example in which the eigenvalues may be complex, we consider the special case in which $(\bar{v}, P(\bar{v}))$ is coincident with the point of local maximum. Then clearly conditions (4.16) will be also necessary for real roots. Besides the case $v_{s}=v_{f}$, this happens for any choice of $v_{s}, v_{f}$, and $h$ when $\varphi$ takes the particular value $\varphi=\frac{1+\gamma}{3+\gamma}, \gamma<1$. In this situation the eigenvalues are complex if and only if $\sqrt{1-2 \varphi}=\sqrt{\frac{1-\gamma}{3+\gamma}}<\frac{\left|v_{s}-v_{f}\right|}{2 \sqrt{g h}}<1$. It is interesting to note that for this particular example we can indeed express the eigenvalues explicitly as $\lambda_{1,4}=$ $\bar{v} \mp \sqrt{B+\sqrt{C}}$, and $\lambda_{2,3}=\bar{v} \mp \sqrt{B-\sqrt{C}}$, where $B=\left(v_{s}-v_{f}\right)^{2} / 4+g h(1-\varphi)$ and $C=g h\left(\left(v_{s}-v_{f}\right)^{2}(1-\varphi)+g h \varphi^{2}\right)$. Therefore, we can check directly the results above.

Proposition 4.1 shows that our model system is hyperbolic for differences of solid and fluid velocities either sufficiently small or sufficiently large, and that there exists a range of values of the phase velocity difference for which the system eigenvalues may be complex and hyperbolicity is lost.

Note that, based on (4.3), the quantities $a=\sqrt{g h}$ and $a \beta=\sqrt{\frac{g}{2} h(1-\varphi)(1-\gamma)}$ appearing in the hyperbolicity conditions represent the propagation speeds of external and internal disturbances, respectively, for flow with $v_{s}=v_{f} \equiv v$, relative to the flow velocity $v$. By considering that $\frac{\left|v_{s}-v_{f}\right|}{2}=\left|v_{s}-\bar{v}\right|=\left|v_{f}-\bar{v}\right|$, where $\bar{v}=\frac{v_{s}+v_{f}}{2}$, we can also interpret (i) of Proposition 4.1 as saying that hyperbolicity holds either if solid and fluid velocities relative to their mean value $\bar{v}$ are both smaller than the characteristic internal speed $a \beta$ of flow in kinematic equilibrium with velocity $\bar{v}$, or these solid and fluid velocities are both larger than the characteristic external speed $a$.

Let us finally mention that the appearance of complex eigenvalues and loss of hyperbolicity that may occur in our model can be analogously observed in the two-layer shallow water model when differences of velocities of the two layers are too large [6,54]. For two-layer flows this phenomenon is related to the appearance of Kelvin-Helmholtz instabilities [6]. For the present two-phase model the occurrence of complex eigenvalues could be related to instabilities that generate strong inhomogeneities in the spatial distribution of the porosity (fluid volume fraction).

\subsection{Eigenvectors}

The right and left eigenvectors of the matrix $A(q)(3.6 \mathrm{~b})$ can be easily written in terms of the eigenvalues $\lambda_{k}, k=1, \ldots, 4$. Let us consider here $\varphi \neq 1$ and $\varphi \neq 0$, that is $h_{s}, h_{f}>0(h>0)$. Then the right eigenvectors $r_{k}, k=1, \ldots, 4$, can be expressed as

$$
r_{k}=\left(1, \lambda_{k}, \xi_{k}, \xi_{k} \lambda_{k}\right)^{\mathrm{T}}
$$

with

$$
\xi_{k}=\frac{\left(\lambda_{k}-v_{s}\right)^{2}-g\left(h_{s}+\frac{1-\gamma}{2} h_{f}\right)}{g \frac{1+\gamma}{2} h_{s}}=\frac{g h_{f}}{\left(\lambda_{k}-v_{f}\right)^{2}-g h_{f}} .
$$

By using the bounds for the eigenvalues in (4.9) and (4.10), one can find that: (i) for $k=1$ and $k=4 \xi_{k}>0$; (ii) for $k=2$ and $k=3$ we have $\xi_{k}<0$ if $\left|v_{s}-v_{f}\right|<2 a \beta, \xi_{k}>0$ if $\left|v_{s}-v_{f}\right|>2 a$, and $\xi_{k}$ can be either positive or negative in the range $2 a \beta \leq\left|v_{s}-v_{f}\right| \leq 2 a$. These results suggest that simple waves associated to the external eigenvalues are characterized by variations of $h_{s}$ and $h_{f}$ and corresponding momenta of the same sign, while variations of opposite sign occur through internal waves in the small phase velocity difference regime.

Let us now assume that the matrix of the right eigenvectors $R=\left(r_{1}, r_{2}, r_{3}, r_{4}\right)$ is non-singular, which is always true if $\left|v_{s}-v_{f}\right|<2 a \beta$ or $\left|v_{s}-v_{f}\right|>2 a$ (Prop. 4.1). Then the left eigenvectors $l_{k}$ of $A(q), k=1, \ldots, 4$, can be taken as

$$
l_{k}=\frac{n_{k}}{P^{\prime}\left(\lambda_{k}\right)}, \quad n_{k}=\left(\vartheta_{s, k}\left(\lambda_{k}-2 v_{s}\right), \vartheta_{s, k}, \vartheta_{f}\left(\lambda_{k}-2 v_{f}\right), \vartheta_{f}\right)
$$


with

$$
\vartheta_{s, k}=\left(\lambda_{k}-v_{f}\right)^{2}-g h_{f}=g \frac{h_{f}}{\xi_{k}} \quad \text { and } \quad \vartheta_{f}=g \frac{1+\gamma}{2} h_{s} .
$$

Here we have normalized the eigenvectors $l_{k}$ so that $L=R^{-1}$, where $L=\left(l_{1}, l_{2}, l_{3}, l_{4}\right)^{\mathrm{T}}$.

Going further into the eigenstructure analysis, we can compute the derivative of the eigenvalues $\lambda_{k}$ along the trajectories of the vector fields $r_{k}$. By evaluating the derivatives $\partial \lambda_{k}(q) / \partial q^{(k)}$ through the characteristic polynomial $P(\lambda)$, we obtain $\nabla \lambda_{k}(q) \cdot r_{k}(q)=\frac{1}{P^{\prime}\left(\lambda_{k}\right)} \Xi_{k}$, with

$$
\Xi_{k}=\frac{2 \vartheta_{s, k}}{h_{s}}\left(\lambda_{k}-v_{s}\right)^{2}+g\left(\vartheta_{s, k}+g \frac{1+\gamma}{2} h_{f}\right)+\xi_{k}\left(\frac{2 \xi_{k} \vartheta_{f}}{h_{f}}\left(\lambda_{k}-v_{f}\right)^{2}+g\left(\xi_{k} \vartheta_{f}+\frac{1-\gamma}{2} \vartheta_{s, k}+\vartheta_{f}\right)\right)
$$

Based on the inequalities written above for $\xi_{k}$, we can verify that $\nabla \lambda_{k}(q) \cdot r_{k}(q) \neq 0 \forall q$ for $k=1$ and $k=4$, that is the external characteristic fields are genuinely nonlinear. On the other hand, the quantity $\nabla \lambda_{k}(q) \cdot r_{k}(q)$ could cross zero for $k=2$ and $k=3$. We leave to the reader to verify these statements.

To conclude this section, we write for completeness the expression of the right eigenvectors for the particular cases omitted above. For $\varphi=1\left(h_{f}=0\right)$, we can still use the normalization (4.17a) (but not the last expression in $(4.17 \mathrm{~b}))$. Here we have $\xi_{k}=0$ for the eigenvalues $v_{s} \mp \sqrt{g h}$, and $\xi_{k}=2\left(\left(v_{s}-v_{f}\right)^{2}-g h\right) /(g h(1+\gamma))$ for the double eigenvalue $v_{f}$. The eigenvalue $v_{f}$ has algebraic multiplicity 2 and geometric multiplicity 1 , and the matrix $R$ is singular (to be precise, note that the algebraic multiplicity of $v_{f}$ becomes 3 in the special situation $\left.\left|v_{s}-v_{f}\right|=\sqrt{g h}\right)$.

For $\varphi=0\left(h_{s}=0\right)$ the right eigenvectors can be written as $\left(\kappa_{k}, \kappa_{k} \lambda_{k}, 1, \lambda_{k}\right)^{\mathrm{T}}$, with $\kappa_{k}=0$ for the eigenvalues $v_{f} \mp \sqrt{g h}$, and with $\kappa_{k}=-\frac{1+\gamma}{2}+\frac{2\left(v_{s}-v_{f}\right)}{g h_{f}}\left(\frac{v_{s}-v_{f}}{2} \mp \sqrt{g h_{f} \frac{1-\gamma}{2}}\right)$ for the eigenvalues $v_{f} \mp \sqrt{g h_{f} \frac{1-\gamma}{2}}$.

\section{A REDUCED MODEL}

Let us assume that drag is strong enough to drive instantaneously the phase velocities to equilibrium. Then we can derive from (3.1) a reduced model describing the dynamics of the two-phase mixture with scaled density $\rho=\varphi+\gamma(1-\varphi)$ and velocity $v \equiv v_{s}=v_{f}$. By adding mass and momentum phase equations, and considering the velocity equilibrium limit, we obtain the following reduced system, here in conservative form:

$$
\begin{aligned}
& \partial_{t} h+\partial_{x}(h v)=0, \\
& \partial_{t}(\rho h)+\partial_{x}(\rho h v)=0, \\
& \partial_{t}(\rho h v)+\partial_{x}\left(\rho h v^{2}+\frac{g}{2} \rho h^{2}\right)=-g \rho h \partial_{x} b-\operatorname{sgn}(v) \nu^{\mathrm{b}} g\left(1-\frac{\gamma}{\rho}\right) \rho h .
\end{aligned}
$$

Note that in this model the volume fraction $\varphi=\frac{\rho-\gamma}{1-\gamma}$, as well as $\rho$, is simply advected:

$$
\partial_{t} \varphi+v \partial_{x} \varphi=0
$$

The analysis of the eigenstructure of the model above is much simpler than the one of the full model (3.1). The system of equations is strictly hyperbolic and it has eigenvalues $\lambda_{1,3}^{\mathrm{r}}=v \mp \sqrt{g h}, \lambda_{2}^{\mathrm{r}}=v$. The second characteristic field corresponds to a contact discontinuity across which $v$ and $\mathcal{P} \equiv \frac{g}{2} \rho h^{2}$ are constant. As we can deduce from equation (5.2), the volume fraction $\varphi$ (equivalently $\rho$ ) can only vary across this contact wave.

Let us now consider the steady state conditions at rest (omitting friction) for the reduced model (5.1). We have

$$
\partial_{x}(h+b)=-\frac{h}{2 \rho} \partial_{x} \rho
$$

We observe that these conditions include those of the full model, equations (3.9), but they also allow equilibrium states with non-uniform $h+b$ and non-uniform $\rho$, hence non-uniform $\varphi$. Since we wish to describe phenomena 
for which physically meaningful equilibrium conditions are only those in (3.9), the reduced model above appears to be physically inappropriate. That is, the hypothesis of instantaneous velocity equilibrium seems physically inconsistent whenever we wish to consider a variable volume fraction. Although the model (5.1) by itself may not be physically interesting, it could be useful for comparison with the full two-phase model, as we will see in Section 10.4.

\section{Numerical solution: a Roe-type method}

We will assume hereafter that $h_{s}, h_{f}>0$ during the flow evolution, and that the difference between solid and fluid velocities is small enough so that the model system is strictly hyperbolic ((i) of Prop. 4.1).

We develop a numerical solution method for system (3.5) in the framework of finite volume schemes based on Riemann solvers (cf. [12,26,50]).

We consider first the solution of system (3.5) without source terms, and we discuss in the following sections the numerical treatment of bottom topography and drag terms. Hence, here we will consider the solution of the homogeneous system

$$
\partial_{t} q+\partial_{x} f(q)+w\left(q, \partial_{x} q\right)=0
$$

with $f(q)$ and $w\left(q, \partial_{x} q\right)$ as in (3.5b), or, in quasi-linear form,

$$
\partial_{t} q+A(q) \partial_{x} q=0
$$

with $A(q)$ as in in (3.6b). Correspondingly, the mixture momentum equation is given by (3.7a) with zero right-hand side.

The difficulties in solving the system above are related to its non-conservative character and the coupling between the equations of the solid and fluid phase. Note that the mass equations of the two materials are in conservation form, together with the mixture momentum equation. On the other hand, the momentum equations contain non-conservative products involving the derivatives of the unknowns that couple the sets of equations of the two constituents. The presence of these coupling terms suggests to avoid trying to solve separately the equations of the two phases through splitting approaches, since uncoupled methods could generate numerical instabilities. Instead, we opt for a global solution technique. See for instance [6] for a discussion on analogous difficulties in the context of two-layer flow modeling, and instabilities produced by uncoupled schemes.

Here we numerically solve our model system by employing a Roe-type method [44], similarly to the approach used in [6] for the two-layer shallow flow model. We consider a computational grid with cells of uniform size $\Delta x$, and we denote with $\Delta t$ the time step. We call $Q_{i}^{n}$ the approximate average solution in the $i$ th cell, $i \in \mathbb{Z}$, at time $t^{n}=n \Delta t, n \in \mathbb{N}$. Following the classical Roe's technique, at every time level $t^{n}$, and at each cell interface $i+1 / 2$ between average solution values $Q_{i}^{n}$ and $Q_{i+1}^{n}$, we solve a Riemann problem for the linearized system

$$
\partial_{t} q+\hat{A}\left(Q_{i}^{n}, Q_{i+1}^{n}\right) \partial_{x} q=0,
$$

with initial data $Q_{i}^{n}$ and $Q_{i+1}^{n}$. The constant coefficient matrix $\hat{A}\left(Q_{i}^{n}, Q_{i+1}^{n}\right)$ is defined so as to guarantee conservation for the mass of each phase and for the momentum of the mixture. That is, we need

$$
\begin{aligned}
f^{(p)}\left(Q_{i+1}^{n}\right)-f^{(p)}\left(Q_{i}^{n}\right) & =\hat{A}^{(p,:)}\left(Q_{i+1}^{n}-Q_{i}^{n}\right), \quad p=1,3, \\
f_{\mathrm{m}}\left(Q_{i+1}^{n}\right)-f_{\mathrm{m}}\left(Q_{i}^{n}\right) & =\left(\hat{A}^{(2,:)}+\gamma \hat{A}^{(4,:)}\right)\left(Q_{i+1}^{n}-Q_{i}^{n}\right),
\end{aligned}
$$

where $f^{(p)}$ denotes the $p$ th component of the vector $f, A^{(p::)}$ the $p$ th row of the matrix $\hat{A}$, and $f_{\mathrm{m}}$ is the flux of the mixture momentum in (3.7b). The conditions (6.4) above can be satisfied by taking $\hat{A}$ as the original 
matrix $A(q)$ evaluated in an average state $\hat{q}=\hat{q}\left(\hat{h}_{s}, \hat{h}_{f}, \hat{v}_{s}, \hat{v}_{f}\right)$, where

$$
\hat{h}_{\theta}=\frac{h_{\theta, i}^{n}+h_{\theta, i+1}^{n}}{2} \quad \text { and } \quad \hat{v}_{\theta}=\frac{\sqrt{h_{\theta, i}^{n}} v_{\theta, i}^{n}+\sqrt{h_{\theta, i+1}^{n}} v_{\theta, i+1}^{n}}{\sqrt{h_{\theta, i}^{n}}+\sqrt{h_{\theta, i+1}^{n}}}, \quad \theta=s, f \text {. }
$$

Let us remark that the mass conservation conditions (6.4a) are indeed identically satisfied for any choice of the average state $\hat{q}$.

\section{F-WAVE FORMULATION}

The Roe-type method we use is formulated in the framework of the wave-propagation algorithms of [24], which are a class of high-resolution finite volume schemes based on Riemann solvers for the solution of hyperbolic systems. In particular, here we adopt a special formulation of these algorithms based on the f-wave method introduced in [3], since this approach will be useful to include topography source terms in the solution of the equations. The f-wave technique is designed for conservative systems, that is, in the homogeneous case, systems of the form $\partial_{t} q+\partial_{x} \mathcal{F}(q)=0$, where $q \in \mathbb{R}^{M}$, and $\mathcal{F}(q) \in \mathbb{R}^{M}$ is a flux function. The key idea is to decompose the flux jump $\Delta \mathcal{F}$ across cell interfaces into a set of $M_{w}$ waves $\mathcal{Z}^{k}$ propagating with some speeds $s^{k}, k=1, \ldots, M_{w}$, that approximate the local Riemann solution. These waves $\mathcal{Z}^{k}$ are called $f$-waves since they correspond to jumps in the flux $\mathcal{F}$. For the interface $i+1 / 2$, and at time $t^{n}$, we have the decomposition

$$
\Delta \mathcal{F} \equiv \mathcal{F}\left(Q_{i+1}^{n}\right)-\mathcal{F}\left(Q_{i}^{n}\right)=\sum_{k=1}^{M_{w}} \mathcal{Z}_{i+1 / 2}^{k} .
$$

F-waves and speeds resulting from local Riemann problems are then used to determine the left-going and right-going fluctuations $\mathcal{A}^{\mp} \Delta Q$ at cell interfaces:

$$
\mathcal{A}^{-} \Delta Q_{i+1 / 2}=\sum_{k: s_{i+1 / 2}^{k}<0} \mathcal{Z}_{i+1 / 2}^{k} \quad \text { and } \quad \mathcal{A}^{+} \Delta Q_{i+1 / 2}=\sum_{k: s_{i+1 / 2}^{k}>0} \mathcal{Z}_{i+1 / 2}^{k}
$$

Finally, the solution is updated at time $n+1$ through the second-order formula:

$$
\begin{aligned}
Q_{i}^{n+1} & =Q_{i}^{n}-\frac{\Delta t}{\Delta x}\left(\mathcal{A}^{+} \Delta Q_{i-1 / 2}+\mathcal{A}^{-} \Delta Q_{i+1 / 2}\right)-\frac{\Delta t}{\Delta x}\left(F_{i+1 / 2}^{\mathrm{c}}-F_{i-1 / 2}^{\mathrm{c}}\right), \\
F_{i+1 / 2}^{\mathrm{c}} & =\frac{1}{2} \sum_{k=1}^{M_{w}} \operatorname{sgn}\left(s_{i+1 / 2}^{k}\right)\left(1-\frac{\Delta t}{\Delta x}\left|s_{i+1 / 2}^{k}\right|\right) \mathcal{Z}_{i+1 / 2}^{c, k},
\end{aligned}
$$

where $F_{i+1 / 2}^{\mathrm{c}}$ are correction fluxes for second order resolution, and $\mathcal{Z}_{i+1 / 2}^{c, k}$ are a modified version of $\mathcal{Z}_{i+1 / 2}^{k}$, obtained by applying to $\mathcal{Z}_{i+1 / 2}^{k}$ a limiter function (see [26] for a more detailed description).

If a Roe linearization $\hat{A}\left(Q_{i}^{n}, Q_{i+1}^{n}\right) \in \mathbb{R}^{M \times M}$ of the Jacobian $\mathcal{F}^{\prime}(q)$ is used to approximate local Riemann problems, then f-waves and associated speeds correspond to the $M$ eigenpairs $\left\{\hat{r}_{k}, \hat{\lambda}_{k}\right\}_{1 \leq k \leq M}$ of $\hat{A}$. In this case $s_{i+1 / 2}^{k}=\hat{\lambda}_{k}$ and $\mathcal{Z}_{i+1 / 2}^{k}=\zeta_{k} \hat{r}_{k}$, where $\zeta_{k}$ are the coefficients of the projection of $\Delta \mathcal{F}$ onto the Roe eigenvectors, $\Delta \mathcal{F}=\sum_{k=1}^{M} \zeta_{k} \hat{r}_{k}$.

In our model system mass equations are conservative, but phase momentum equations contain non-conservative products. Therefore we lack a flux vector $\mathcal{F}$ to be used for a wave decomposition of the form (7.1). Nonetheless, we can still formulate our Roe-type scheme into the structure of the algorithm above, if we define locally an approximate flux difference $\Delta \tilde{\mathcal{F}}$ to be splitted into Roe eigencomponents in a way that is consistent with 
the Roe linearization $\hat{A}$ of our system. Here we define $\Delta \tilde{\mathcal{F}}$ as the sum of the jump in the flux vector $f$ of the conservative portion of the equations (see $(3.5 \mathrm{~b})$ ), plus a contribution resulting from a linearization of the nonconservative term $w\left(q, \partial_{x} q\right)$ corresponding to the Roe system. Based on the local approximation of $w\left(q, \partial_{x} q\right)$ as $\left(0, \gamma g \hat{h}_{s} \partial_{x} h_{f}, 0, g \hat{h}_{f} \partial_{x} h_{s}\right)^{\mathrm{T}}$, where $\hat{h}_{s}, \hat{h}_{f}$ are the Roe averages in (6.5), we take:

$$
\Delta \tilde{\mathcal{F}}=\Delta f+\left(\begin{array}{c}
0 \\
\gamma g \hat{h}_{s} \Delta h_{f} \\
0 \\
g \hat{h}_{f} \Delta h_{s}
\end{array}\right)
$$

where $\Delta f=f\left(Q_{i+1}^{n}\right)-f\left(Q_{i}^{n}\right)$, and $\Delta h_{\theta}=h_{\theta, i+1}^{n}-h_{\theta, i}^{n}, \theta=s, f$. Note that $\Delta \tilde{\mathcal{F}}=\hat{A} \Delta q, \Delta q \equiv Q_{i+1}^{n}-Q_{i}^{n}$. Hence, by construction of $\hat{A}$, we see that $\Delta \tilde{\mathcal{F}}$ satisfies the mixture momentum conservation condition $\Delta \tilde{\mathcal{F}}^{(2)}+\gamma \Delta \tilde{\mathcal{F}}^{(4)}=$ $f_{\mathrm{m}}\left(Q_{i+1}^{n}\right)-f_{\mathrm{m}}\left(Q_{i}^{n}\right)$.

Denoting here with $\left\{\hat{r}_{k}, \hat{\lambda}_{k}\right\}_{1 \leq k \leq 4}$ the eigenpairs of the Roe matrix $\hat{A}$ of our system, we then use the decomposition

$$
\Delta \tilde{\mathcal{F}}=\sum_{k=1}^{4} \zeta_{k} \hat{r}_{k}
$$

and we define f-waves and corresponding speeds as

$$
\mathcal{Z}_{i+1 / 2}^{k}=\zeta_{k} \hat{r}_{k} \quad \text { and } \quad s_{i+1 / 2}^{k}=\hat{\lambda}_{k}, \quad k=1, \ldots, 4 .
$$

These quantities are employed to update cell averages through (7.2)-(7.3). Based on the property $\Delta \tilde{f}=\hat{A} \Delta q$, note that the $\mathrm{f}$-waves defined above are exactly the flux jumps of the solution of the Roe linear system, given by $\hat{\lambda}_{k} \alpha_{k} \hat{r}_{k}$, where $\alpha_{k}$ are the coefficients of the eigen-decomposition $\Delta q=\sum_{k=1}^{4} \alpha_{k} \hat{r}_{k}$, and we have the equivalence $\zeta_{k}=\hat{\lambda}_{k} \alpha_{k}, k=1, \ldots, 4$.

\section{TOPOGRAPHY SOURCE TERMS}

We now consider the solution of the model system with the bottom topography source term $\psi^{\mathrm{b}}(q)$ in $(3.5 \mathrm{c})$ included:

$$
\partial_{t} q+A(q) \partial_{x} q=\psi^{\mathrm{b}}(q)
$$

As mentioned in the Introduction, topography terms must be discretized in a way to ensure well-balancing, that is the property of the method of preserving steady state conditions and modeling efficiently small perturbations from them. In particular, for the system under study we are concerned with the steady state conditions at rest in (3.9).

To build a well-balanced scheme, we follow the approach of [3,28], which uses the f-wave formulation framework described in the previous section. Such a technique has been used for instance in the context of tsunami modeling to treat bathymetry source terms [27]. The idea is to incorporate the effect of topography source terms into the Riemann solution, by taking discrete interface values $\Psi_{i+1 / 2}^{\mathrm{b}, n}$ of $\psi^{\mathrm{b}}(q)$, and by including a contribution $\Psi_{i+1 / 2}^{\mathrm{b}, n} \Delta x$ into the splitting of $\Delta \tilde{\mathcal{F}}$ in (7.5). In place of (7.5), we now decompose

$$
\Delta \tilde{\mathcal{F}}-\Psi_{i+1 / 2}^{\mathrm{b}, n} \Delta x=\sum_{k=1}^{4} \zeta_{k} \hat{r}_{k} .
$$

F-waves $\mathcal{Z}_{i+1 / 2}^{k}=\zeta_{k} \hat{r}_{k}$ obtained from this splitting, together with their propagating speeds $s_{i+1 / 2}^{k}=\hat{\lambda}_{k}$, are then used to compute the fluctuations at interfaces as in (7.2), and the solution is updated through (7.3). 
The motivation for this approach is that if cell averages $Q_{i}^{n}$ and interface source terms $\Psi_{i+1 / 2}^{\mathrm{b}, n}$ satisfy at time $t^{n}$ the discrete steady state condition

$$
\frac{\Delta \tilde{\mathcal{F}}}{\Delta x}=\Psi_{i+1 / 2}^{\mathrm{b}, n}
$$

then the left-hand side of (8.2) will be zero, therefore $\zeta^{k}=0, \forall k=1, \ldots, 4$. Hence, all the f-waves $\mathcal{Z}_{i+1 / 2}^{k}$ will have zero strength, and the wave-propagation algorithm (7.2)-(7.3) will give $Q_{i}^{n+1}=Q_{i}^{n}$. That is, the discrete steady state is maintained by this method. If the solution does not satisfy (8.3) exactly but is close to a steady state, then (8.2) means that it is the deviation from steady state that is used to determine the $\mathcal{Z}_{i+1 / 2}^{k}[28]$.

The interface source term $\Psi_{i+1 / 2}^{\mathrm{b}, n}$ must be defined so that the discrete steady state condition (8.3) holds whenever initial Riemann data correspond to equilibrium, that is when $(h+b)_{i}^{n}=(h+b)_{i+1}^{n}, \varphi_{i}^{n}=\varphi_{i+1}^{n}$, $v_{s, i}^{n}=v_{s, i+1}^{n}=v_{f, i}^{n}=v_{f, i+1}^{n}=0$. To satisfy this requirement we take

$$
\Psi_{i+1 / 2}^{\mathrm{b}, n} \Delta x=-\left(\begin{array}{c}
0 \\
g \hat{h}_{s} \Delta b \\
0 \\
g \hat{h}_{f} \Delta b
\end{array}\right),
$$

with $\Delta b=b_{i+1}-b_{i}$.

\section{DRAG SOURCE TERMS}

We illustrate in this section the numerical treatment of the drag term $\psi^{\mathrm{D}}(q)$ in $(3.5 \mathrm{c})$ for the solution of the complete model system $(3.5)$. Note that $\psi^{\mathrm{D}}(q)$ vanishes at steady states at rest, hence it does not influence the steady state equilibrium conditions (3.9). Based on this, there is no special need for well-balancing techniques for this term, and we employ a simple fractional step method for its numerical approximation. Here we alternate between solving over a time step $\Delta t$ the model system without drag (see previous sections), and solving over a time step $\Delta t$ a system of ordinary differential equations for the drag contribution:

$$
\partial_{t} q=\psi^{\mathrm{D}}(q)
$$

In particular, for the numerical solution of this system we will adopt the approach used in [36,37] in the context of dusty gas modeling.

We recall that in $(3.5 \mathrm{c}) D=\frac{1}{\rho_{f}} \mathcal{D}\left(\varphi,\left|v_{f}-v_{s}\right| ; \sigma\right)$, where $\sigma$ is a vector of constant physical parameters (e.g. the solid particle diameter). Based on drag relations proposed in the literature for multiphase granular materials, e.g. $[55,56]$ and reviews $[32,51]$, here we consider functions $\mathcal{D}$ in the following quasi-linear form in $\left|v_{f}-v_{s}\right|:$

$$
\mathcal{D}=S_{1}(\varphi, \Sigma(R e) ; \sigma)+S_{2}(\varphi, \Sigma(R e) ; \sigma)\left|v_{f}-v_{s}\right|,
$$

where $\Sigma(R e)$ is a vector of physical parameters (e.g. the drag coefficient) that may depend on $\left|v_{f}-v_{s}\right|$ through the Reynolds number of the flow

$$
R e=\frac{(1-\varphi) \rho_{f} d_{s}\left|v_{f}-v_{s}\right|}{\mu_{f}} .
$$

Here $d_{s}$ is the solid particle diameter, and $\mu_{f}$ is the dynamic viscosity of the fluid. The general drag relation above encompasses also the drag function used by Pitman and Le, which we reported in Section 2. For another example of $\mathcal{D}$, see Section 10.4. 
By using (9.2) the system of ODEs in (9.1) takes the form

$$
\begin{aligned}
& \partial_{t} h_{s}=0, \\
& \partial_{t}\left(h_{s} v_{s}\right)=\frac{1}{\rho_{s}}\left(h_{s}+h_{f}\right)\left(S_{1}+S_{2}\left|v_{f}-v_{s}\right|\right)\left(v_{f}-v_{s}\right), \\
& \partial_{t} h_{f}=0, \\
& \partial_{t}\left(h_{f} v_{f}\right)=-\frac{1}{\rho_{f}}\left(S_{1}+S_{2}\left|v_{f}-v_{s}\right|\right)\left(h_{s}+h_{f}\right)\left(v_{f}-v_{s}\right),
\end{aligned}
$$

with initial data $h_{s}^{0},\left(h_{s} v_{s}\right)^{0}, h_{f}^{0},\left(h_{f} v_{f}\right)^{0}$ coming from the hyperbolic solver. Here and in the following the superscript 0 indicates initial values. From (9.4a) and (9.4c) we immediately see that the variables $h_{f}$ and $h_{s}$ are constant over $\Delta t$ :

$$
h_{f}(\Delta t)=h_{f}^{0} \quad \text { and } \quad h_{s}(\Delta t)=h_{s}^{0} .
$$

Each step we assume that the parameters $\Sigma(R e)$ are constant in time, and equal to their initial values $\Sigma^{0}=$ $\Sigma\left(R e^{0}\right)$. It follows that the functions $S_{1,2}$ are also constant, and equal to $S_{1,2}^{0}=\left(\varphi^{0}, \Sigma^{0} ; \sigma\right)$, where $\varphi^{0}=\frac{h s^{0}}{h_{s}^{0}+h_{f}^{0}}$. Then the solution of the momentum equations (9.4b), (9.4d) can be found exactly as

$$
\left(h_{s} v_{s}\right)(\Delta t)=\left(h_{s} v_{s}\right)^{0}-\gamma \frac{h^{0} \Delta v^{0}}{\varsigma^{0}}\left(\frac{S_{1}^{0}}{\left(S_{1}^{0}+\left|\Delta v^{0}\right| S_{2}^{0}\right) e^{\varsigma^{0} \frac{S_{1}^{0}}{\rho_{f}} \Delta t}-\left|\Delta v^{0}\right| S_{2}^{0}}-1\right),
$$

and

$$
\left(h_{f} v_{f}\right)(\Delta t)=\left(h_{f} v_{f}\right)^{0}+\frac{h^{0} \Delta v^{0}}{\varsigma^{0}}\left(\frac{S_{1}^{0}}{\left(S_{1}^{0}+\left|\Delta v^{0}\right| S_{2}^{0}\right) e^{\varsigma^{0} \frac{S_{1}^{0}}{\rho_{f}} \Delta t}-\left|\Delta v^{0}\right| S_{2}^{0}}-1\right)
$$

where $h=h_{s}+h_{f}$, and where we have introduced the quantities

$$
\Delta v=v_{f}-v_{s} \quad \text { and } \quad \varsigma=\left(h_{s}+h_{f}\right)\left(\frac{1}{h_{f}}+\frac{\gamma}{h_{s}}\right)=\frac{1}{1-\varphi}+\frac{\gamma}{\varphi} .
$$

In the limit case $S_{1} \equiv 0$ the solution for the momenta has the form

$$
\left(h_{s} v_{s}\right)(\Delta t)=\left(h_{s} v_{s}\right)^{0}-\gamma \frac{h^{0} \Delta v^{0}}{\varsigma^{0}}\left(\frac{1}{\frac{S_{2}^{0}}{\rho_{f}} \varsigma^{0}\left|\Delta v^{0}\right| \Delta t+1}-1\right),
$$

and

$$
\left(h_{f} v_{f}\right)(\Delta t)=\left(h_{f} v_{f}\right)^{0}+\frac{h^{0} \Delta v^{0}}{\varsigma^{0}}\left(\frac{1}{\frac{S_{2}^{0}}{\rho_{f}} \varsigma^{0}\left|\Delta v^{0}\right| \Delta t+1}-1\right) .
$$

If instead $S_{2} \equiv 0$, note that the last term between parentheses on the right of (9.6) simplifies to $\left(\mathrm{e}^{-\varsigma^{0} \frac{S_{1}^{0}}{\rho_{f}} \Delta t}-1\right)$.

We then use formulas (9.5), (9.6) ((9.8) if $\left.S_{1} \equiv 0\right)$ for the numerical solution of the system of ODEs for the drag term. The advantage of this semi-exact algorithm is that it is efficient for both fast and slow velocity relaxation processes. If the time scale for reaching phase velocity equilibrium is much smaller than the time scale corresponding to the hyperbolic wave-propagation behavior of the system, then the source term is stiff, 
and other explicit ODEs solvers may require for stability a time step too small to be used in practice. We refer for instance to [26] for a discussion on numerical difficulties with stiff source terms in hyperbolic systems.

Let us finally remark that if the drag relaxation time is small relative to the time step, then the solid and fluid velocities as determined from the updating equations (9.6) or (9.8) both approach the common equilibrium value

$$
v_{\mathrm{eq}}=\frac{h_{s}^{0} v_{s}^{0}+\gamma h_{f}^{0} v_{f}^{0}}{h_{s}^{0}+\gamma h_{f}^{0}}
$$

\section{Numerical EXPERIMENTS}

\subsection{Implementation and eigenvalues computation}

The numerical scheme that we have presented in the previous sections has been implemented on the basis of the Fortran 77 routines of the CLAWPACK software [23].

As we have seen, the method requires the computation of the eigenstructure of the Roe matrix $\hat{A}$. We recall that simple explicit formulas for the system's eigenvalues are not available except in the special case $v_{s}=v_{f}$ (Sect. 4). Here we use Newton's iteration method to find the external Roe eigenvalues $\hat{\lambda}_{1,4}$, employing as initial guess the bounds in (4.4) for the roots of the characteristic polynomial. More precisely, we use starting guess $v_{\min }-\sqrt{g \hat{h}}$ to find $\hat{\lambda}_{1}$, and starting guess $v_{\max }+\sqrt{g \hat{h}}$ to obtain $\hat{\lambda}_{4}$, where $\hat{h}=\hat{h}_{s}+\hat{h}_{f}, v_{\min }=\min \left(\hat{v}_{f}, \hat{v}_{s}\right)$ and $v_{\max }=\max \left(\hat{v}_{f}, \hat{v}_{s}\right)$. Typically convergence to the roots is fast. The only situation in which root finding can be slow is when the average solid volume fraction $\hat{\varphi}=\frac{\hat{h}_{s}}{\hat{h}}$ is very close to 1 , in which case the derivative of the characteristic polynomial may be close to zero in the neighborhood of one of the external roots. In this case one could switch to a bisection method with a choice of the starting interval based on (4.9). Knowing $\hat{\lambda}_{1,4}$, we can then obtain the internal Roe eigenvalues $\hat{\lambda}_{2,3}$ by simply using Vieta's formulas, which relate roots and coefficients of polynomials. Once we have all the $\hat{\lambda}_{k}, k=1, \ldots, 4$, the right and left Roe eigenvectors are immediately found through the relations (4.17) and (4.18), respectively.

As a final remark, let us remind that it is well known that Roe's solver may fail when the Roe eigenvalues are close to sonic points. To overcome this problem, a technique based on the Harten and Hyman entropy-fix [17] has been applied.

\subsection{Tests with flat bottom and with no drag}

We present in this subsection some numerical tests over flat bottom surface and with no drag contribution, that is we take $b(x)=0$ and $D=0$. In all the experiments we set $g=9.8$ and $\gamma=1 / 2$. Moreover, we apply free flow boundary conditions, and we use CFL number $=0.9\left(\mathrm{CFL}=\frac{\Delta t}{\Delta x} \max _{i}\left(\max _{1 \leq k \leq 4}\left|\hat{\lambda}_{k}\right|\right) i\right.$, where $i \in \mathbb{Z}$ is the grid cell index).

\subsubsection{Initial flow hump with higher fluid content}

We perform a numerical experiment that has been suggested to us by a test for single-phase shallow water equations in [26] (p. 257) that shows the evolution of an initial hump of water. Here we add to the problem in [26] a perturbation of the solid volume fraction, and we observe the waves arising from a flow hump that contains a higher fluid content with respect to the surrounding region.

Initially, the fluid is at rest $\left(v_{s}=v_{f}=0\right)$ and the profiles of the flow depth and of the solid volume fraction are defined by the following Gaussian functions over the domain $[-15,15]$ :

$$
h=h_{0}+\delta \mathrm{e}^{-16 x^{2}} \quad \text { and } \quad \varphi=\varphi_{0}-\delta \mathrm{e}^{-16 x^{2}},
$$

with $h_{0}=1, \varphi_{0}=0.6$ and $\delta=0.2$ or $\delta=0.2 \times 10^{-3}$.

To begin with, we consider a small perturbation with respect to the state characterized by $h=h_{0}$ and $\varphi=\varphi_{0}$, and we take $\delta=0.2 \times 10^{-3}$. In this case we expect that the solution of the problem consists 
approximately of four linear waves propagating at the characteristic speeds corresponding to the background state, that is $\lambda_{1,4}=\mp \sqrt{g h_{0}}$ for the external waves, and $\lambda_{2,3}=\mp \sqrt{\frac{g}{2} h_{0}\left(1-\varphi_{0}\right)(1-\gamma)}$ for the internal waves. We perform the computation with 1000 grid cells, and with the monotonized centered (MC) limiter for second order correction terms.

Figure 2 shows the results for $h, \varphi$, and $v_{s}, v_{f}$ at several times. The dotted line in the plots of $h$ and $\varphi$ represents the initial profile of these variables. As predicted, we see that the initial perturbation splits into four waves that then move with their profile unchanged. The numerical speed of the waves corresponds to the expected values $\lambda_{1,4}=\mp 3.1305$ and $\lambda_{2,3}=\mp 0.9899$.

Note that the perturbation of $\varphi$ induces a variation of the phase velocity difference $\left(v_{s}-v_{f}\right)$, consistently with the equation governing $\left(v_{s}-v_{f}\right)$ in our model, which is, with $b(x)=0$ and $D=0$,

$$
\partial_{t}\left(v_{s}-v_{f}\right)+\frac{g h}{2 \varphi}(1-\gamma) \partial_{x} \varphi+\frac{1}{2} \partial_{x}\left(v_{s}^{2}-v_{f}^{2}\right)=0
$$

We can observe that in this problem $\varphi$ and $\left(v_{s}-v_{f}\right)$ vary only across the internal waves. To try to understand this behavior, we can compute the derivative of $\varphi$ along the direction of the characteristic fields. We obtain, for $k=1, \ldots, 4$ :

$$
\nabla \varphi \cdot r_{k}=\frac{h_{s}}{\left(h_{s}+h_{f}\right)^{2}}\left(\frac{h_{f}}{h_{s}}-\xi_{k}\right)
$$

with $\xi_{k}$ as in (4.17b). If $v_{s}=v_{f}$, then for the external characteristic fields $(k=1$ and $k=4)$ we have $\xi_{k}=h_{f} / h_{s}$, therefore the derivative above is zero. It is then reasonable to expect small variations of $\varphi$ across external waves when phase velocities are close. Although this provides a partial explanation, as we will see in the next experiment (Fig. 3), also for larger initial perturbations of $\varphi$, and hence larger induced variations of $\left(v_{s}-v_{f}\right)$, the same phenomenon occurs. Moreover, numerical tests of Riemann problems show that no variation of $\varphi$ and $\left(v_{s}-v_{f}\right)$ may take place across rarefactions and even shocks associated to the external characteristic fields, when at initial time $v_{s}=v_{f}$ (e.g. experiment in the next subsection, Fig. 5). To fully understand such behavior, detailed knowledge of the Riemann solution structure would be needed, including the Riemann invariants, but unfortunately we lack an analytical description of it.

We now repeat the numerical test above with a larger variation of $h$ and $\varphi$, using here the initial profiles (10.1) with $\delta=0.2$. Results are displayed in Figure 3. In this case we can clearly observe the effects of nonlinearities, which cause the steepening of the front of the waves.

Finally, to appreciate the single influence of variations of flow depth and solid volume fraction, we plot in Figure 4 the solution at time $t=3.5$ for the cases in which we set: (a) only an initial perturbation of $h$, (b) only an initial perturbation of $\varphi$, in both cases with $\delta=0.2$.

For case (a) we see that the solid volume fraction $\varphi$ and the phase velocity difference remain unperturbed. This is in general the behavior of the solution of any problem for which we have initial conditions $\varphi=$ const. and $v_{s}=v_{f}$. In this case $\varphi=$ const. and $v_{s}=v_{f}$ at any later time since there are no forces that can induce variations of $\varphi$ or $\left(v_{s}-v_{f}\right)$, and the solution for the flow depth $h$ and the flow velocity $v_{s}=v_{f}$ simply corresponds to the solution of single-phase shallow water equations, whichever is the constant value of $\varphi$. Figure 4 a shows that the flow hump generates two gravity waves moving in opposite directions, as in the shallow water example of [26], p. 257.

In Figure $4 \mathrm{~b}$ we see that the initial variation of $\varphi$ produces perturbations in all the variables and causes the formation of four waves, analogously to the problem of Figure 3 (see Fig. 3d at time $t=3.5$ ). Note that here (Fig. 4b) the increase of $h$ induced by $\varphi$ in correspondence of the internal waves is balanced by a decrease of $h$ through the external waves as required by mass conservation. 

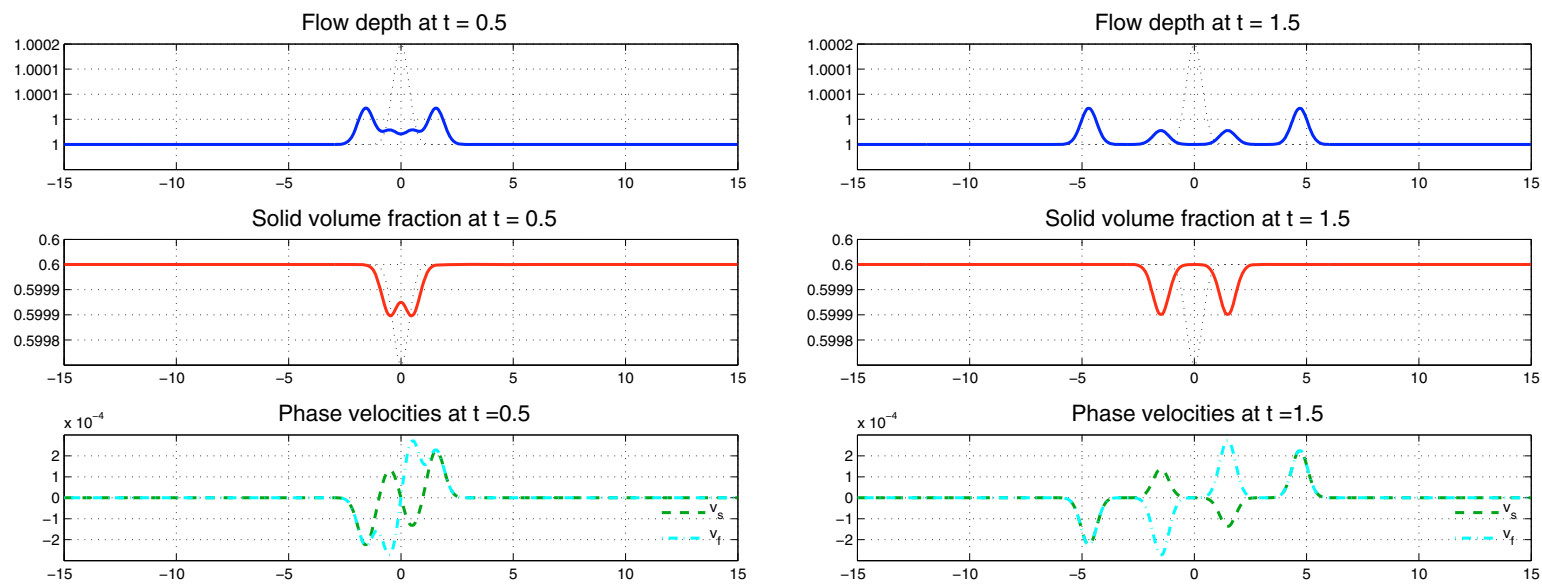

(a) $t=0.5$

(b) $t=1.5$
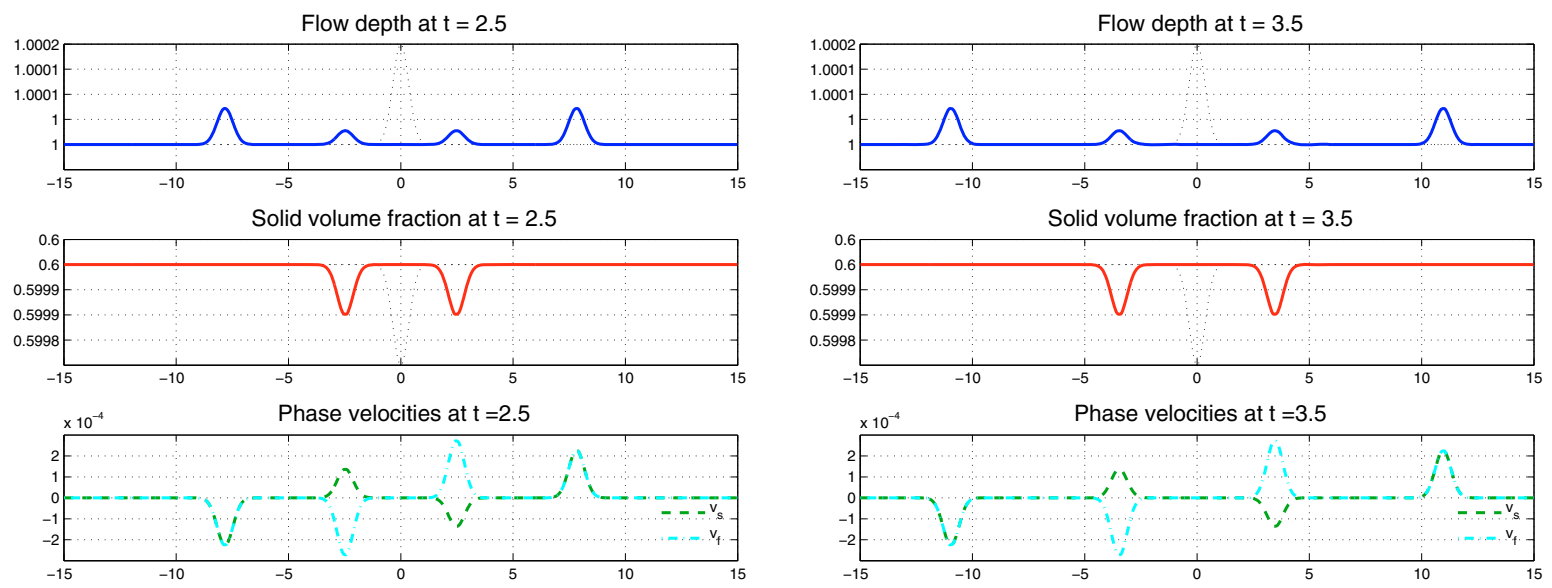

(d) $t=3.5$

(c) $t=2.5$

Figure 2. Waves generated by a small perturbation of the flow depth and of the solid volume fraction, $\delta=0.2 \times 10^{-3}$. Each sub-figure shows the flow depth $h$, the solid volume fraction $\varphi$, and the phase velocities $v_{s}$ (dashed line) and $v_{f}$ (dash-dot line). The dotted line in the plots of $h$ and $\varphi$ represents the initial profiles.

\subsubsection{Riemann problems}

We solve here two Riemann problems, for which the initial conditions consist of two constant states separated by an interface located at $x=0$. We define the initial values of the flow height and of the solid volume fraction as

$$
h=3, \quad \varphi=0.7 \quad \text { if } x<0 \quad \text { and } \quad h=2, \quad \varphi=0.4 \quad \text { if } x>0 .
$$

In the first problem we assume the fluid initially at rest on both sides on the interface (dam-break problem), i.e. $v_{s}=v_{f}=0$ everywhere in the domain. We perform the computation over the interval $[-5,5]$ with 100 and 1000 grid cells, employing the Minmod limiter for second order corrections.

Results at time $t=0.5$ are displayed in Figure 5. Figures $5 \mathrm{a}, 5 \mathrm{~b}$ and $5 \mathrm{~d}$ compare the results obtained with the two different grid resolutions for the flow depth variables, the solid volume fraction, and the phase velocities. In these plots the solid line represents the solution computed with the finer grid of 1000 grid cells. 

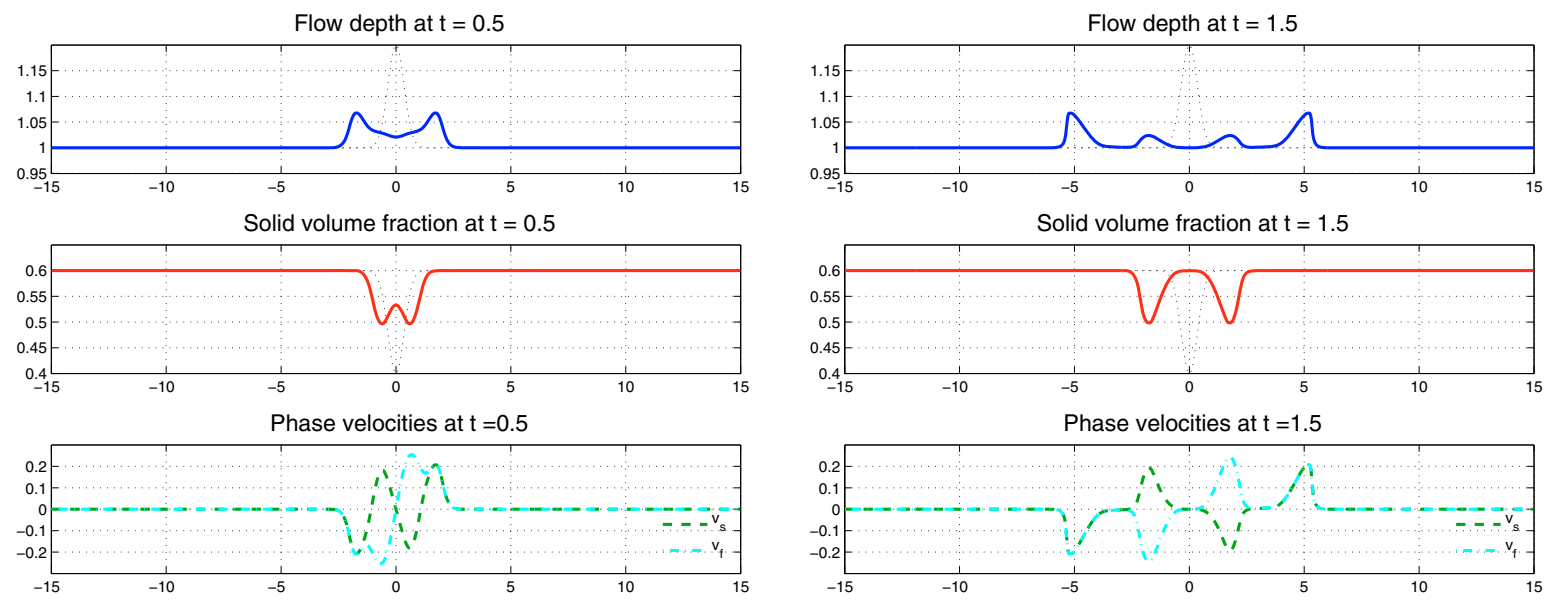

(a) $t=0.5$

(b) $t=1.5$
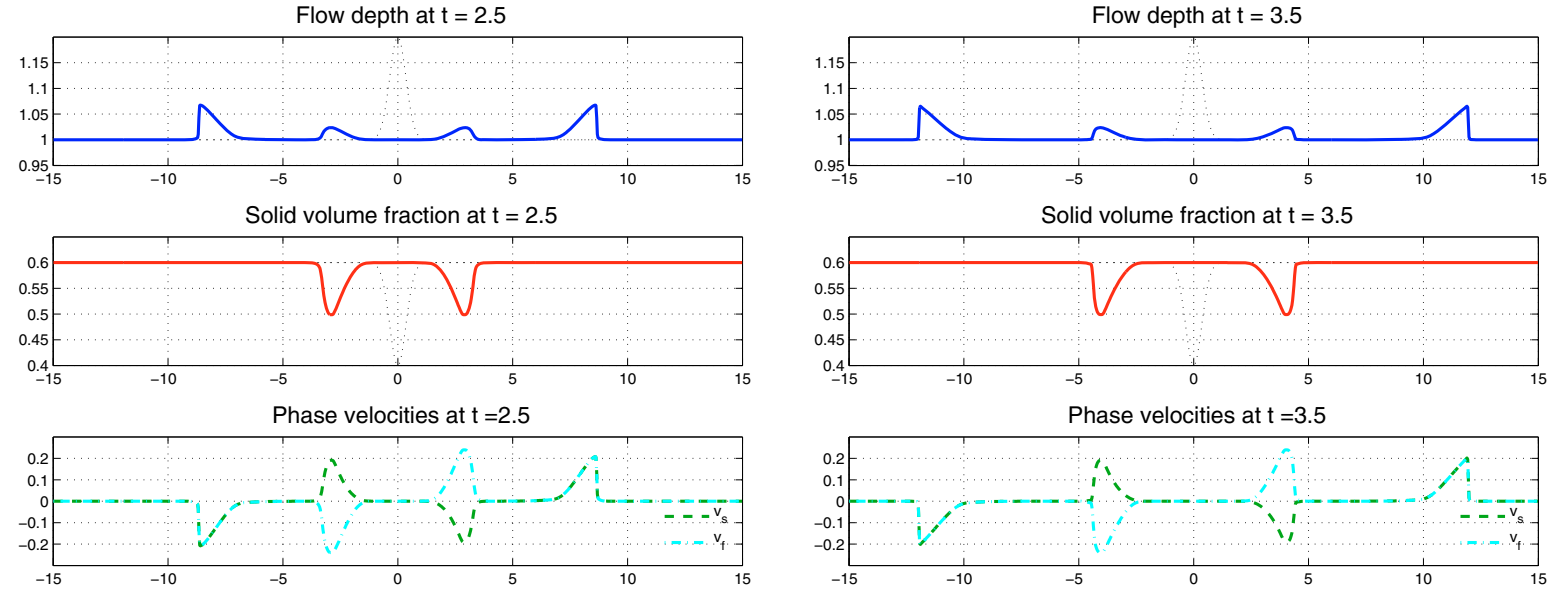

(c) $t=2.5$

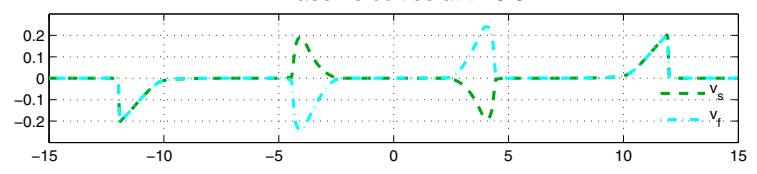

(d) $t=3.5$

FIGURE 3. Waves generated by a perturbation of flow depth and solid volume fraction, $\delta=0.2$.

See caption of Figure 2 for plot description.

Moreover, the dashed line indicates the initial conditions. In Figure 5c we display the eigenvalues as computed with the finer grid.

We can observe that the Riemann solution consists of a 1-rarefaction, a 2-shock, a 3-rarefaction, and a 4-shock. Note that the eigenvalues $\lambda_{1}$ and $\lambda_{3}$ are monotonically increasing along the rarefactions of the associated characteristic fields. As anticipated in the previous subsection, we can see that $\varphi$ appears to vary only across the internal waves.

We now solve a Riemann problem with the same initial data for $h$ and $\varphi$ in (10.4), but we assume here an initial phase velocity difference, taking

$$
v_{s}=-1.4, \quad v_{f}=0.3 \quad \text { if } x<0 \quad \text { and } \quad v_{s}=-0.9, \quad v_{f}=-0.1 \quad \text { if } x>0 \text {. }
$$

Results are displayed in Figure 6. The solution of this Riemann problem, as in the previous case, is made of a 1-rarefaction, a 2-shock, a 3-rarefaction, and a 4-shock. However, in this case we can observe that the initial phase velocity difference induces a variation of $\varphi$ across the 1-rarefaction and the 4-shock. 

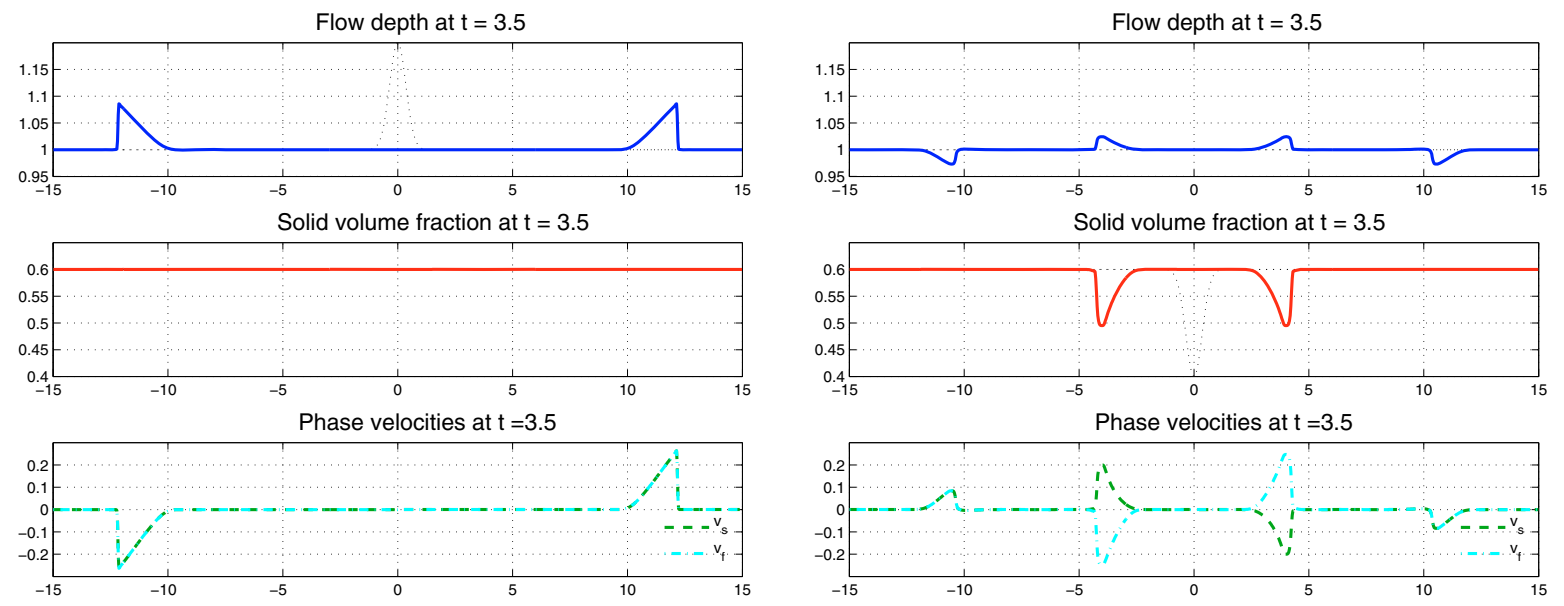

(a)

(b)

FiguRE 4. (a) Waves generated by a perturbation of the flow depth. (b) Waves generated by a perturbation of the solid volume fraction. $\delta=0.2, t=3.5$. See caption of Figure 2 for plot description.

Finally, for both the two Riemann problems presented we plot the ratio $N=\frac{\left|v_{s}-v_{f}\right|}{2 a \beta}$ to show the fulfillment of the hyperbolicity condition $N<1$ of Proposition 4.1, and to observe how far we are in these particular examples from the limit $N=1$ of the hyperbolicity region. Note that, based on the observations at the end of Section 4.1, the dimensionless number $N$ can be interpreted as the ratio between the deviation of solid and fluid velocities from their mean value $\left|v_{s}-\bar{v}\right|=\left|v_{f}-\bar{v}\right|=\frac{\left|v_{s}-v_{f}\right|}{2}$ and the characteristic propagation speed $a \beta$ of internal disturbances that would be observed for flow with $v_{s}=v_{f}=\bar{v}$. In Figure 7 we see that $N<1$ for the two problems. Let us mention that our Fortran program would stop in case complex eigenvalues were found, since the model would lose its validity.

\subsection{Tests with variable topography}

We present results of some experiments over variable bottom topography chosen to test the well-balance property of our numerical scheme. For all the problems we use $\gamma=1 / 2, \mathrm{CFL}=0.9$, and the MC limiter.

\subsubsection{Perturbation of a steady state at rest}

We perform an experiment that is an extension of LeVeque's classical test [25] for single-phase shallow water equations with bottom topography. In this problem we observe the behavior of a small perturbation of steady state conditions at rest (3.9) over a bottom topography defined as

$$
b(x)= \begin{cases}0.25(\cos (\pi(x-0.5) / 0.1)+1) & \text { if }|x-0.5|<0.1 \\ 0 & \text { otherwise }\end{cases}
$$

Initially, we take a small perturbation of the flow depth $h$ and of the solid volume fraction $\varphi$ :

$$
h(x, 0)=h_{0}+\tilde{h} \quad \text { and } \quad \varphi(x, 0)=\varphi_{0}-\tilde{\varphi}, \quad \text { for }-0.6<x<-0.5,
$$

with $h_{0}=1, \varphi_{0}=0.6$, and $\tilde{h}=\tilde{\varphi}=10^{-3}$. Figure 8 shows the type of the initial data for the total height $h+b$ and the solid volume fraction $\varphi$, though with much larger $\tilde{h}$ and $\tilde{\varphi}$ for clarity of the plot. The computational domain is $[-0.9,1.1]$, and free flow boundary conditions are used. Moreover, we take $g=1$ and $\gamma=1 / 2$. 


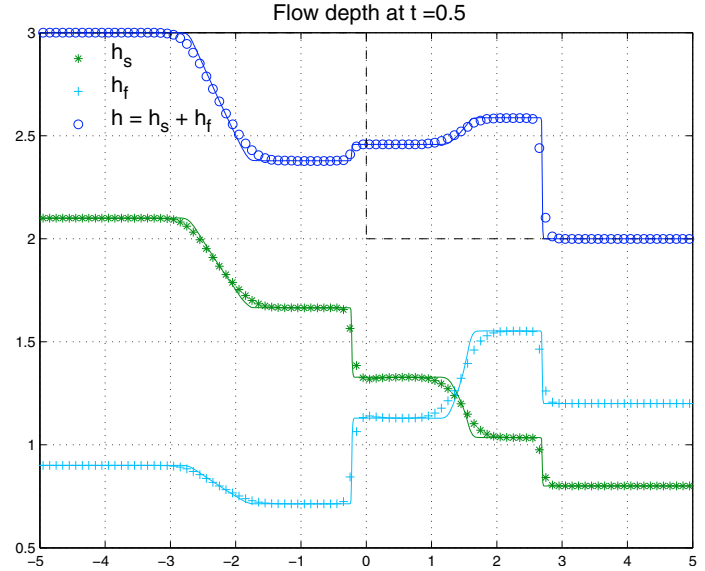

(a)

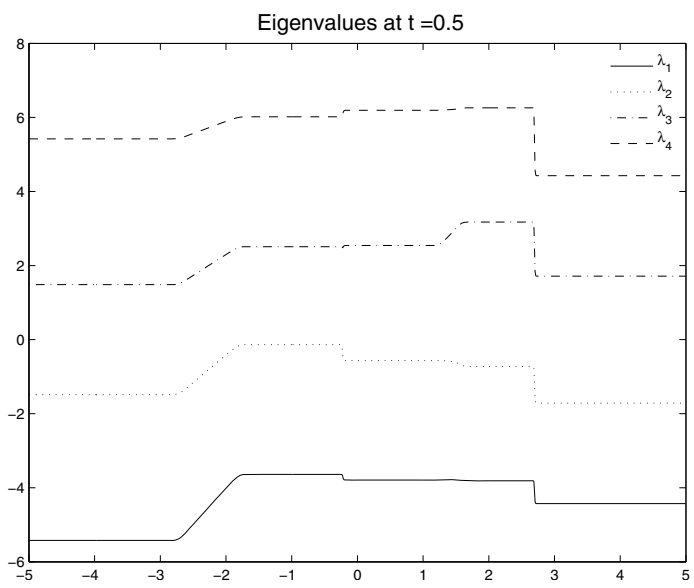

(c)

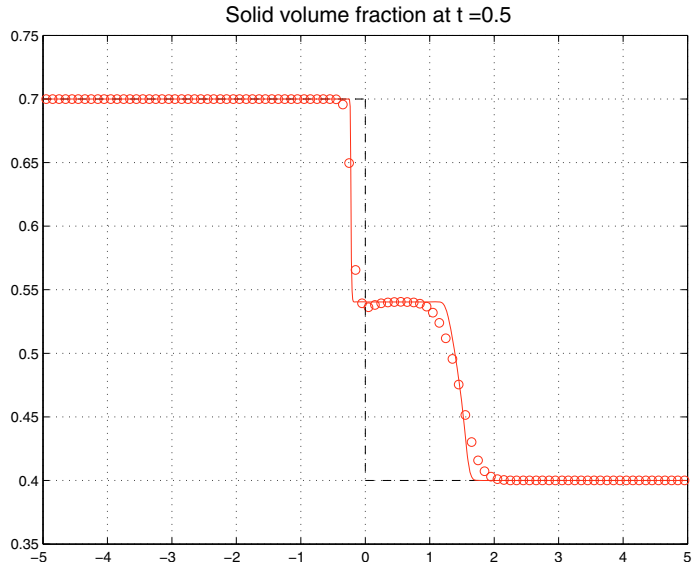

(b)

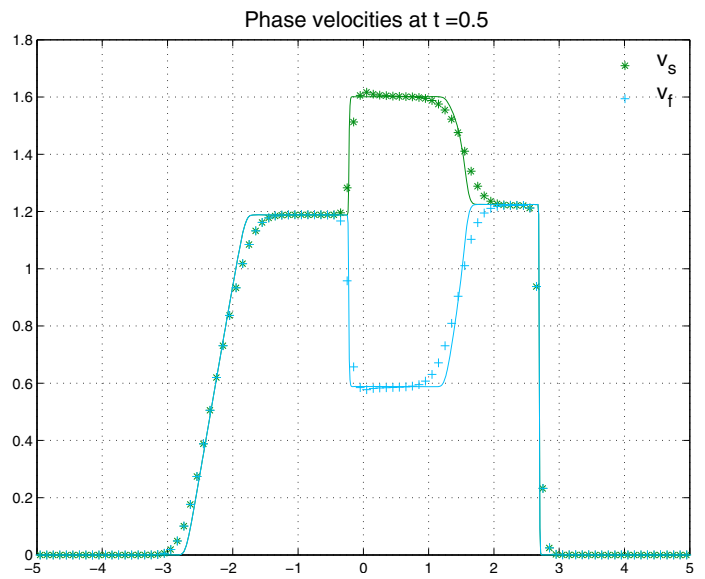

(d)

Figure 5. Dam-break problem. (a), (b) and (d): Results computed with 100 grid cells compared with reference solution obtained with 1000 cells (solid line for every variable). Dashed line: initial conditions. (a) Flow depth $h$ (circles) and variables $h_{s}$ (stars) and $h_{f}$ (pluses); (b) solid volume fraction $\varphi$; (d) phase velocities $v_{s}$ (stars) and $v_{f}$ (pluses). (c) Eigenvalues computed with 1000 grid cells.

We compute the solution with 100 grid cells and compare it with a fine grid reference solution obtained with 1000 grid cells.

In Figure 9 we display the results at six different times for $h+b$ and $\varphi$ (top and bottom subplot of each sub-figure, respectively). The bold line over the $x$-interval $[0.4,0.6]$ in the plots of $h+b$ indicates the region of the domain where $b(x) \neq 0$.

As we can observe from the first sub-figure the initial perturbation splits into four waves, two right-going waves, and two left-going waves that leave the domain from the left edge. Figure 9b shows the time at which the right-going external wave has just passed over the obstacle at the bottom, and it has been partially reflected. In Figure 9c this wave has left the domain from the right, and the reflected wave generated by it has passed through the incoming right-going internal wave. In Figure 9d this internal wave has moved past the hump and has produced its own reflected wave, which can be clearly distinguished in Figure 9e. The last sub-figure shows 


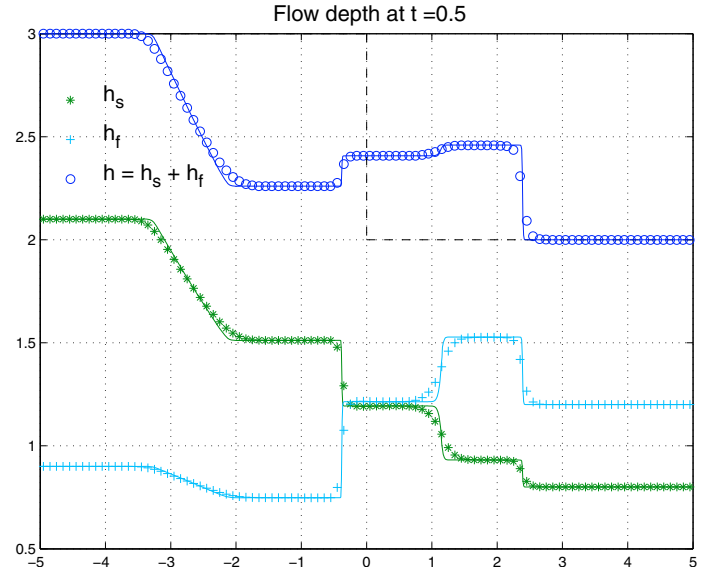

(a)

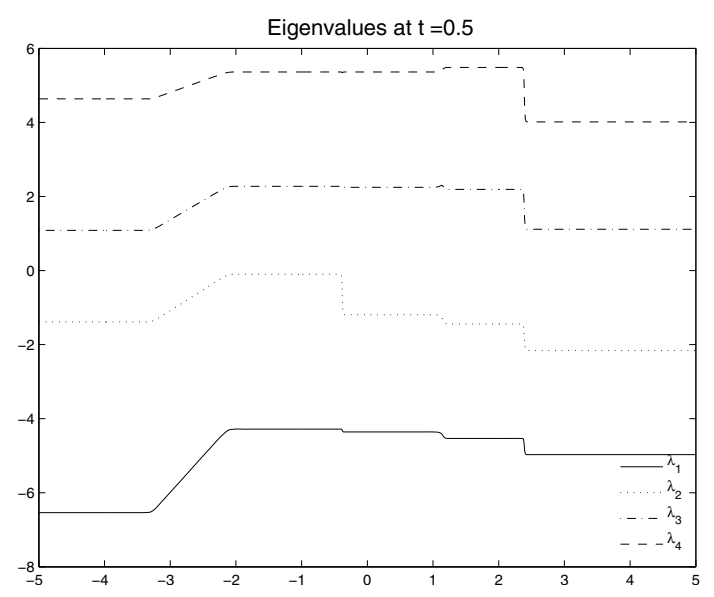

(c)

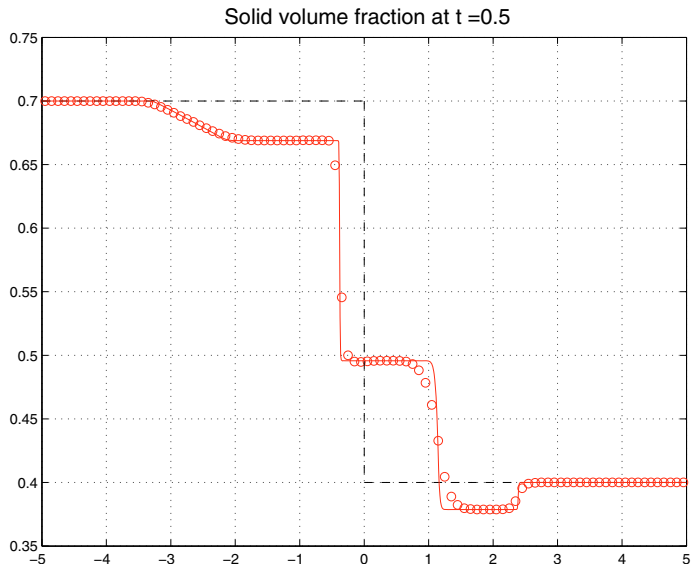

(b)

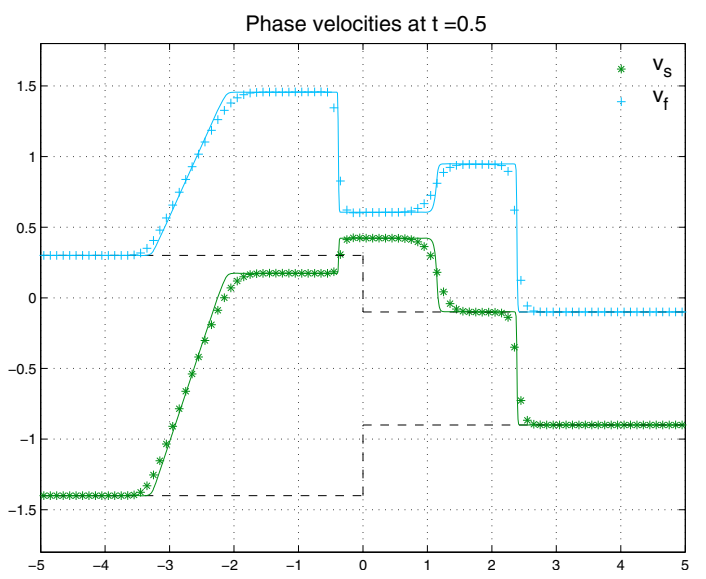

(d)

FIGURE 6. Riemann problem with phase velocity difference in the initial data. See caption of Figure 5 for plot description.

finally the situation in which all the disturbances have exited from the computational region and equilibrium is attained.

It is well known that numerical schemes that are not well-balanced may produce in this kind of numerical tests unphysical disturbances [25]. Our numerical method is able to capture the physically correct reflected waves, without producing spurious oscillations.

\subsubsection{Perturbation of a steady flow in motion}

As we have seen in Section 8, our numerical scheme has been specifically designed to ensure well-balancing for steady state conditions at rest. Nonetheless, we present here an experiment that shows that our method is capable to preserve equilibrium conditions and to model efficiently perturbations from equilibrium also for a steady flow that is in motion.

Here we are concerned with the general equilibrium conditions for a moving steady flow characterized by $v_{s}=v_{f} \equiv v$ :

$$
\varphi=\text { const. }, \quad h v=\text { const. } \quad \text { and } \quad g(h+b)+\frac{1}{2} v^{2}=\text { const. }
$$



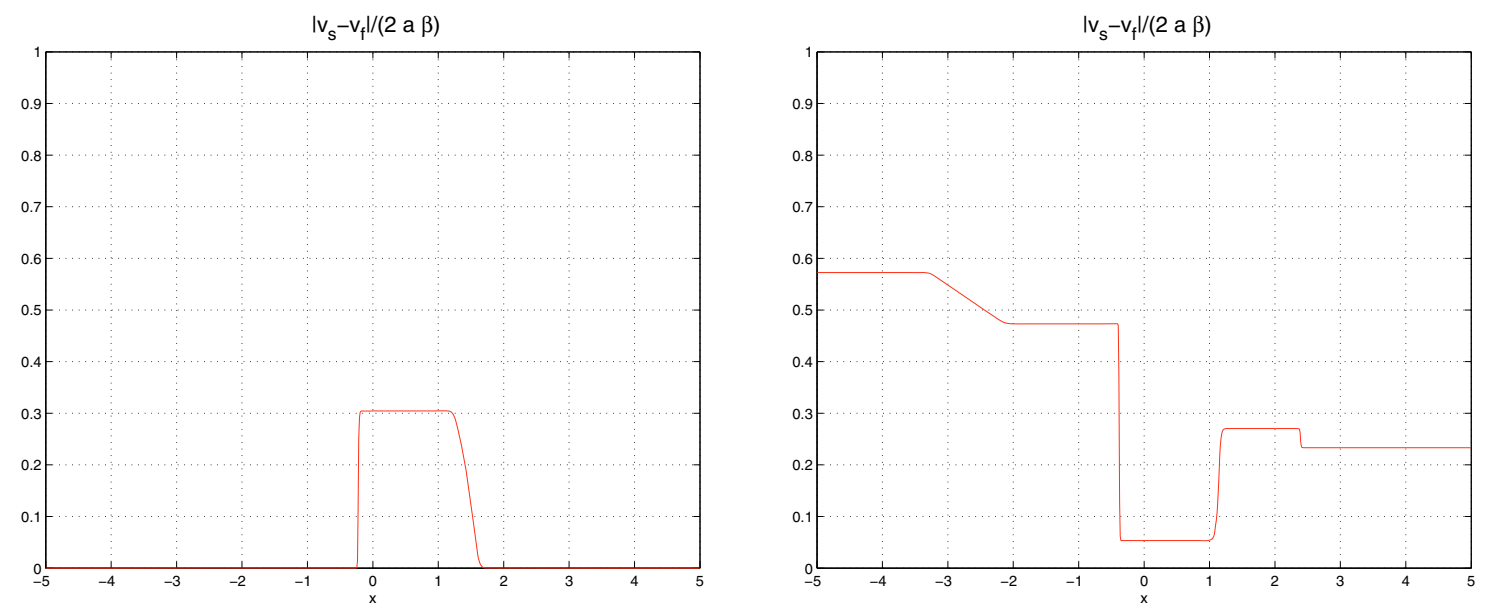

FigURE 7. Ratio $\frac{\left|v_{s}-v_{f}\right|}{2 a \beta}$ at $t=0.5$ for the Riemann problems of Figure 5 (left) and Figure 6 (right).
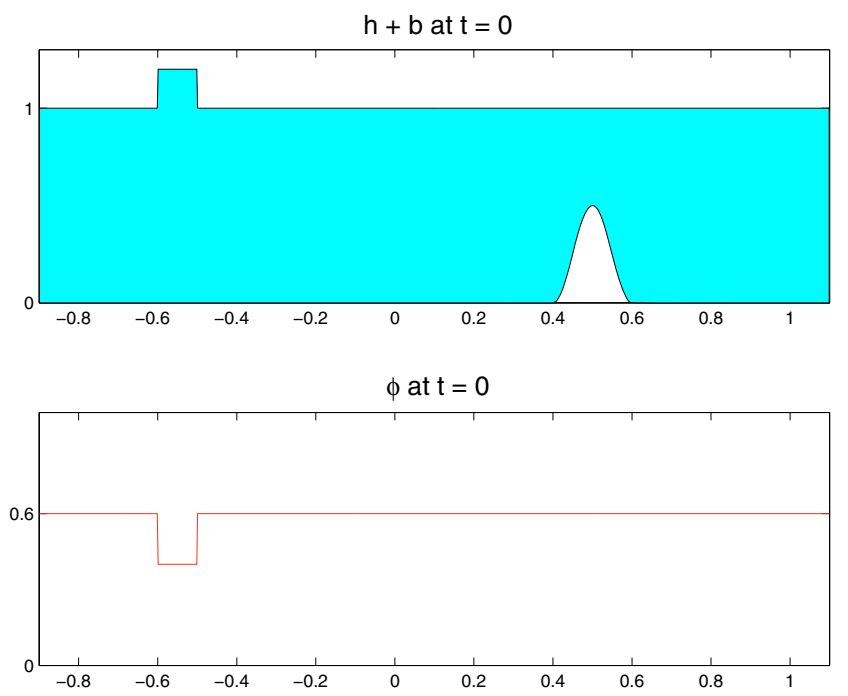

FiguRE 8. Type of initial conditions for the numerical test of subsection 10.3.1. Above: total flow height $h+b$, below: solid volume fraction $\varphi$. (Here $\tilde{h}$ and $\tilde{\varphi}$ are much larger than the values used in the experiment.)

To begin with, we perform an experiment proposed in [14] and then used by several authors (e.g. [10]) to test convergence of classical shallow water equations to a steady subcritical flow moving over a bump. The bottom surface is specified over the interval $[-5,25]$ as

$$
b(x)= \begin{cases}0.2-0.05(x-10)^{2} & \text { if } 8<x<12 \\ 0 & \text { otherwise }\end{cases}
$$

A constant flux $(h v)_{\text {in }}=4.42$ is imposed at the left boundary (inlet), and a flow height $h_{\text {out }}=2$ is fixed at the right boundary (outlet). Initially, we take constant $\varphi$, zero phase velocities, and $h=2$ everywhere in the domain. Moreover, we set $g=9.8$. The computed solution converges to the correct steady state, as we can 


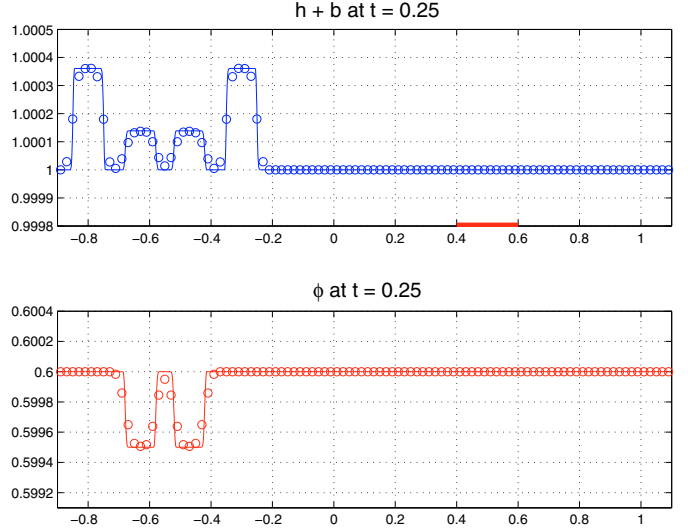

(a) $t=0.25$
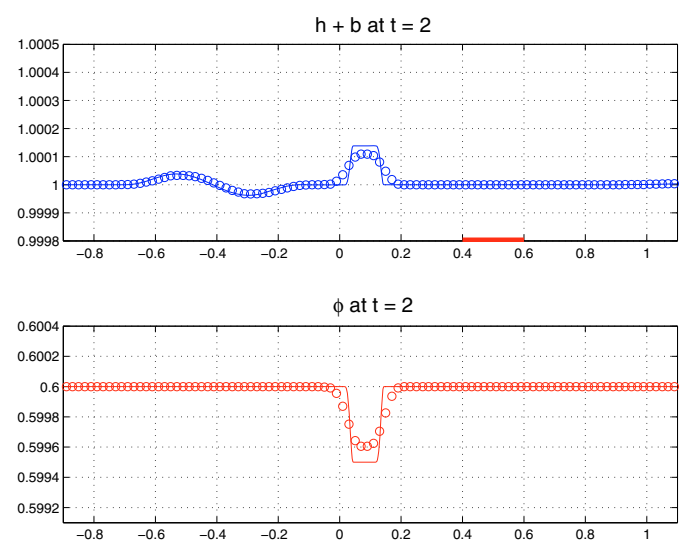

(c) $t=2$
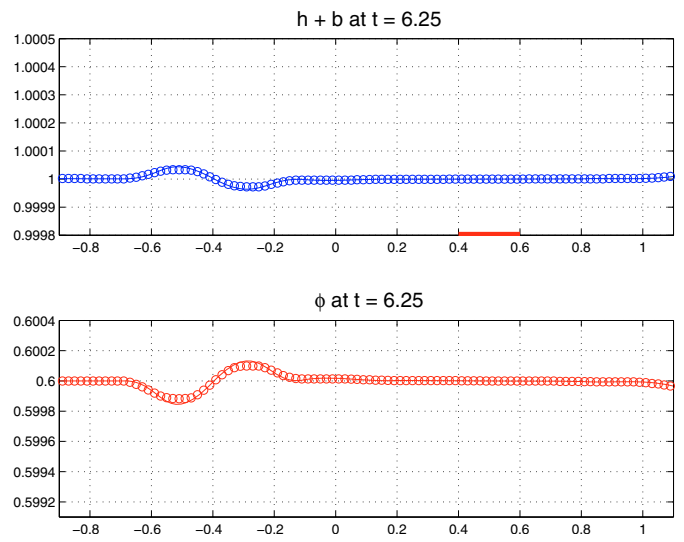

(e) $t=6.25$
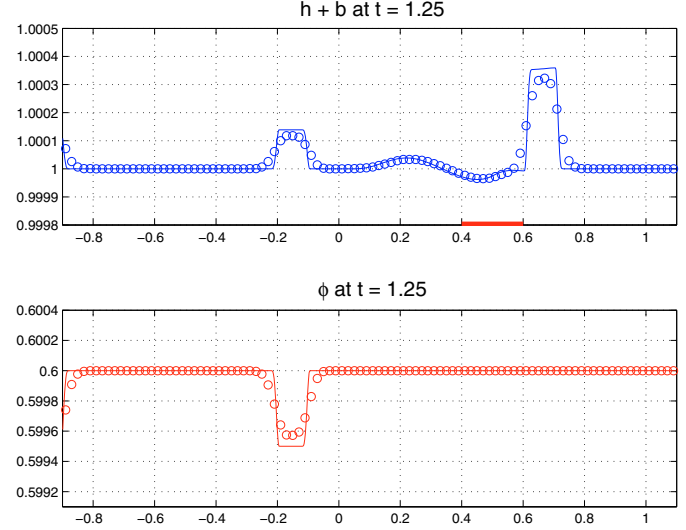

(b) $t=1.25$
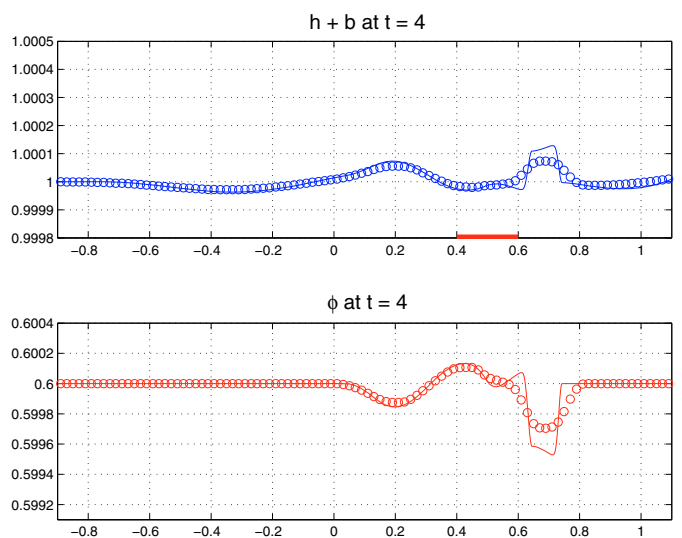

(d) $t=4$
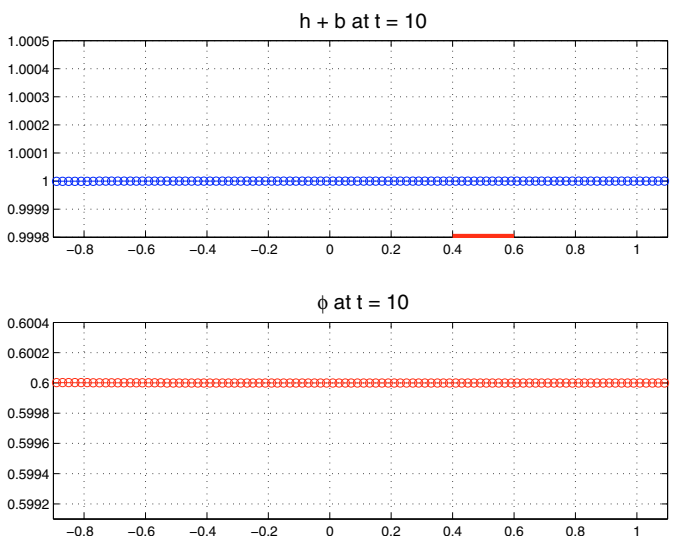

(f) $t=10$

Figure 9. Perturbation of a steady state at rest $\left(\tilde{h}=\tilde{\varphi}=10^{-3}\right)$. Circles: solution computed with 100 grid cells; solid line: reference solution computed with 1000 grid cells. Bold line over $0.4<x<0.6$ : region where $b(x) \neq 0$. 


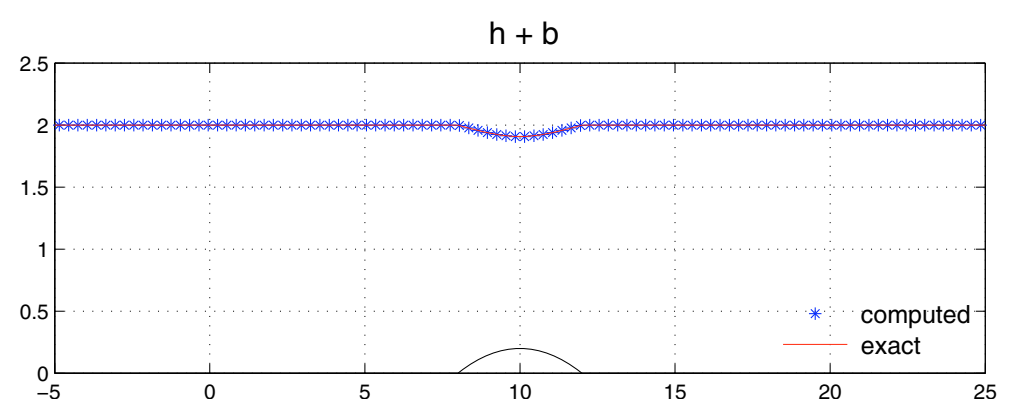

FIGURE 10. Steady subcritical flow over a bump. Analytical solution (solid line) and solution computed with 100 grid cells at $t=50$ (stars).

observe from Figure 10, where we plot results of $h+b$ obtained with 100 grid cells at $t=50$ together with the analytical solution of the problem (the results are superimposed).

Now, we take a small initial perturbation of the steady conditions, and we observe its evolution in time. We set the initial phase velocities and the initial flow height equal to their equilibrium values, while we introduce an initial disturbance for the solid volume fraction, by defining

$$
\varphi(x, 0)=\varphi_{0}+\tilde{\varphi}, \quad \text { for }-3.5 \leq x \leq-2.5
$$

with $\varphi_{0}=0.6$ and $\tilde{\varphi}=10^{-3}$. We perform the experiment with 150 grid cells, and compare the results with a reference solution obtained with 1500 cells. Free flow boundary conditions are used in this test.

Results are displayed in Figure 11 at several times. For each sub-figure, we show the deviation of $h$ from the steady state value $h_{\text {steady }}$, and the solid volume fraction $\varphi$. The bold line over the $x$-interval $[8,12]$ in the subplots of $h-h_{\text {steady }}$ indicates the location of the obstacle at the bottom.

The initial perturbation generates four waves, one of which leaves the domain from the left boundary. Figures $11 \mathrm{a}$ and $11 \mathrm{~b}$ show the passage of the right external wave and of the right internal wave over the bump, respectively, and in Figure 11c we can clearly notice the reflected wave generated by the internal wave. Figure $11 \mathrm{~d}$ shows then the time at which the second internal wave goes over the bump. Finally all the disturbances leave the domain and steady state conditions are attained in the computational region, as we can see from the last plots. As for the test illustrated in Figure 9, no spurious oscillations are generated by the numerical method in this problem.

\subsection{Tests with interphase drag}

We repeat here the flow hump experiment and the dam-break problem of Section 10.2, including now interphase drag effects. We express the drag function $\mathcal{D}$ in (9.2) based of the drag correlation of Gidaspow [11]:

$$
\mathcal{D}= \begin{cases}150 \frac{\varphi^{2} \mu_{f}}{(1-\varphi) d_{s}^{2}}+\frac{7}{4} \frac{\varphi \rho_{f}}{d_{s}}\left|v_{f}-v_{s}\right| & \text { if } \varphi>0.2, \\ \frac{3}{4} c_{d}(R e) \frac{(1-\varphi) \varphi \rho_{f}}{d_{s}}(1-\varphi)^{-2.65}\left|v_{f}-v_{s}\right| & \text { if } \varphi \leq 0.2,\end{cases}
$$

where the drag coefficient $\mathrm{c}_{\mathrm{d}}=\mathrm{c}_{\mathrm{d}}(R e)$ is given by

$$
c_{\mathrm{d}}= \begin{cases}\frac{24}{R e}\left(1+0.15 R e^{0.687}\right) & \text { if } R e<1000 \\ 0.44 & \text { if } R e \geq 1000\end{cases}
$$



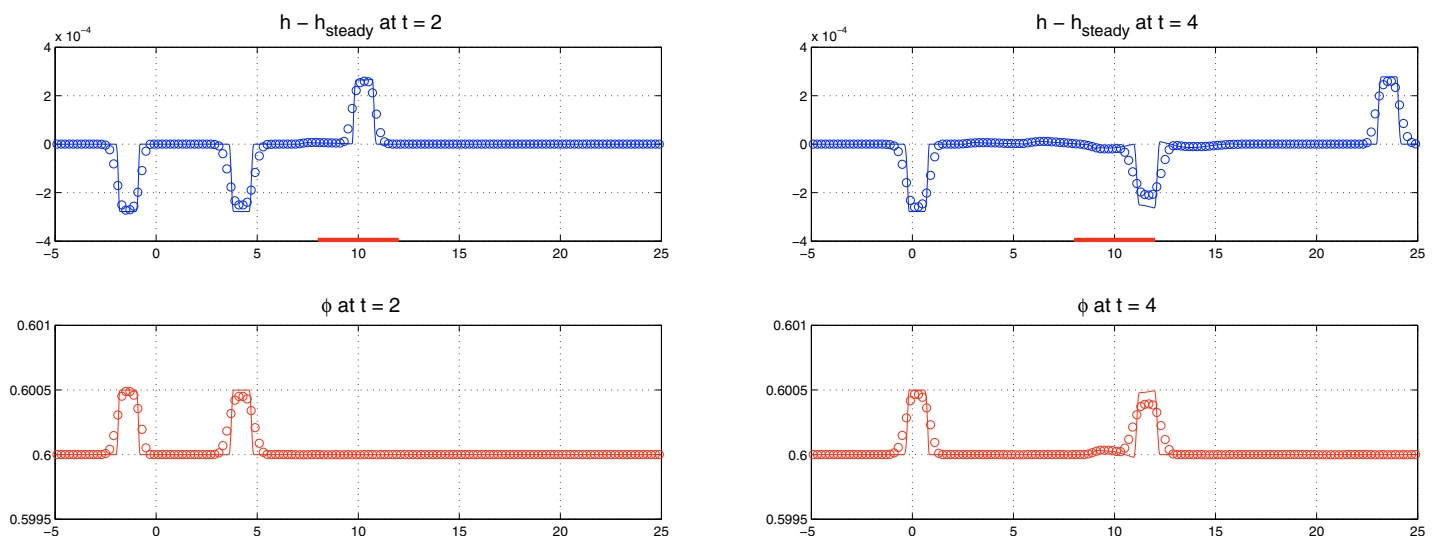

(a) $t=2$

(b) $t=4$
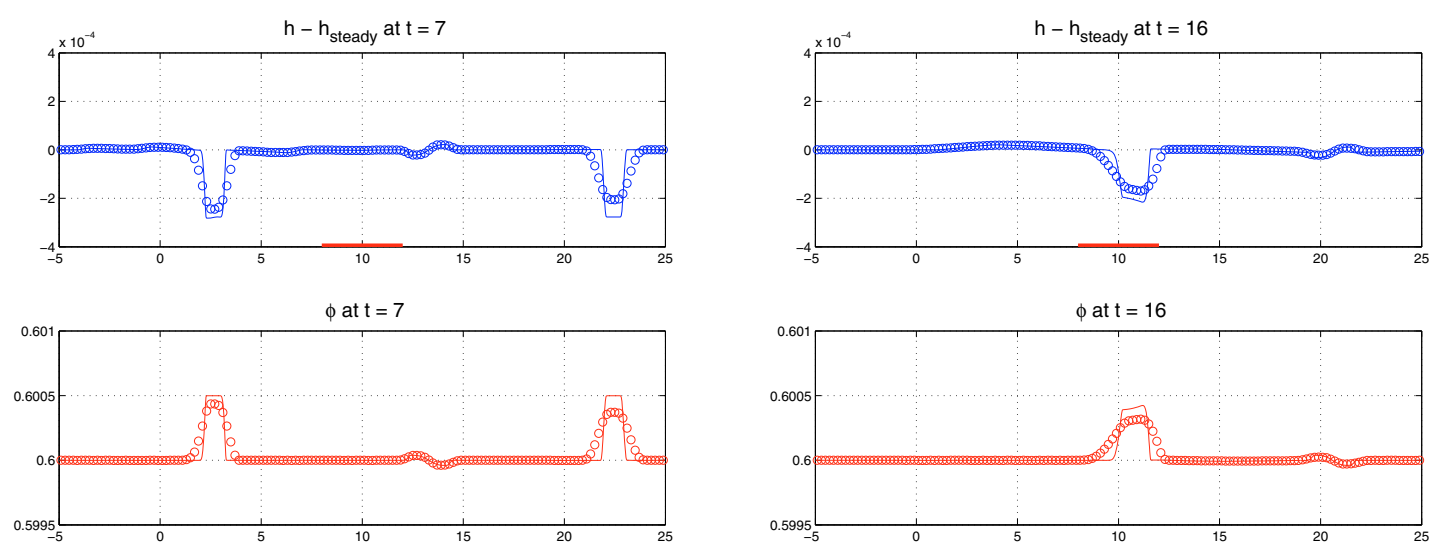

(c) $t=7$

(d) $t=16$
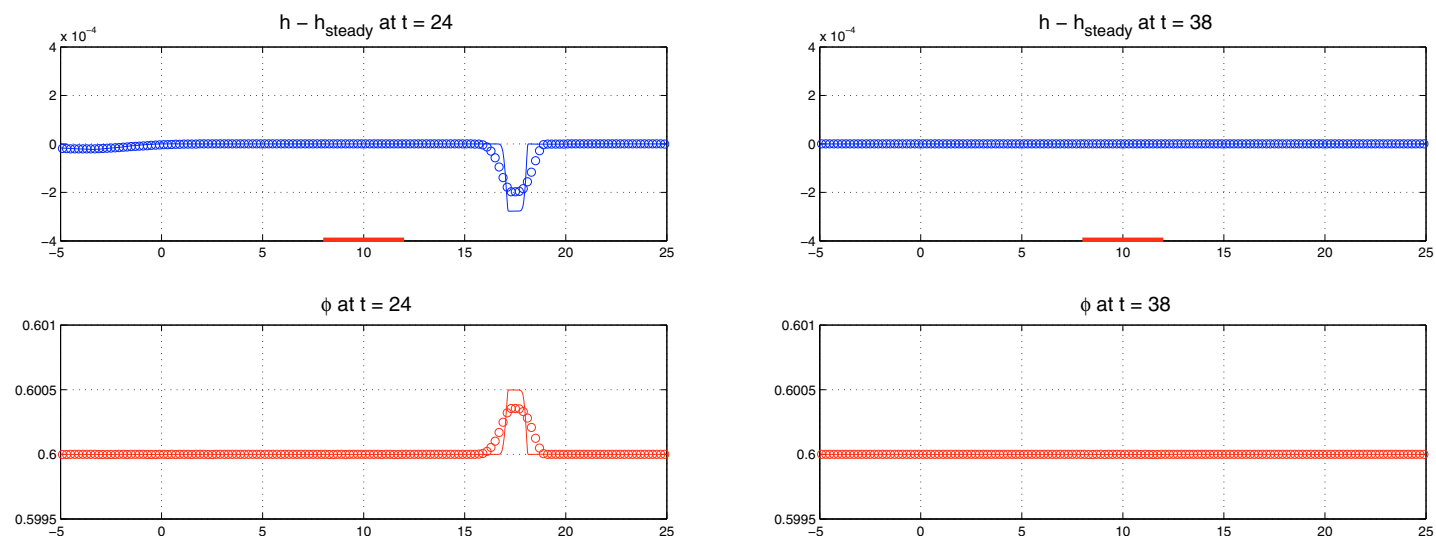

(e) $t=24$

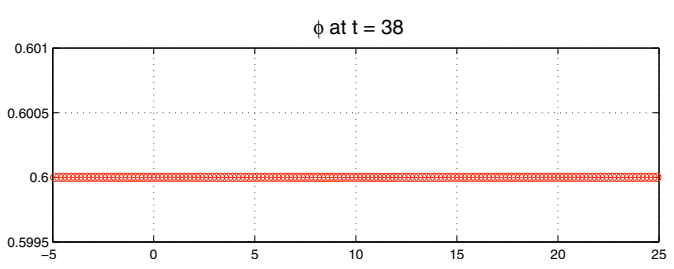

(f) $t=38$

Figure 11. Perturbation of a steady flow moving over a bump $\left(\tilde{\varphi}=10^{-3}\right)$. Circles: solution computed with 150 grid cells; solid line: reference solution computed with 1500 cells. Bold line over $8<x<12$ : region where $b(x) \neq 0$. 
We define the specific densities of the two material as $\rho_{s}=2000 \mathrm{~kg} \cdot \mathrm{m}^{-3}$ and $\rho_{f}=1000 \mathrm{~kg} \cdot \mathrm{m}^{-3}$, so that $\gamma=\rho_{f} / \rho_{s}=1 / 2$ as in the previous experiments. Moreover, we set $d_{s}=0.05 \mathrm{~m}$ and $\mu_{f}=0.001 \mathrm{~Pa} \cdot \mathrm{s}$. Let us remark that it is beyond the scope of the present work to discuss the definition of a physically appropriate drag law for realistic flows. Here we choose a reasonable drag function that allows us to test our numerical scheme and to observe qualitatively the effects of interphase drag forces.

In addition to perform the computations with a definite drag, by employing the algorithm described in Section 9, for each numerical test we also compute the solution of our two-phase system obtained by imposing numerically instantaneous phase velocity equilibrium, as if drag forces were infinitely large. This is done by setting each time step the values of the solid and fluid velocities equal to the equilibrium value $v_{\text {eq }}$ in (9.9). That is, in the fractional step algorithm we replace the updating formulas (9.6) or (9.8) with

$$
\left(h_{s} v_{s}\right)(\Delta t)=h_{s}^{0} v_{\mathrm{eq}} \quad \text { and } \quad\left(h_{f} v_{f}\right)(\Delta t)=h_{f}^{0} v_{\mathrm{eq}} .
$$

Furthermore, we also solve with the same initial data the reduced system (5.1) that was theoretically derived by assuming instantaneous velocity relaxation. We will see that in the limit of infinitely large drag solutions of the two-phase model approach those of the reduced model.

The reduced system is solved numerically by a finite volume scheme that uses a Riemann solver based on Suliciu's relaxation method $[47,48]$. See the Appendix. Let us note, however, that Riemann problems for the reduced model can be solved exactly, thanks to the simplicity of the analysis of the system's eigenstructure.

\subsubsection{Flow hump with drag}

We first compute the solution of our two-phase system with drag terms included for the flow hump experiment of Section 10.2.1, taking $\delta=0.2$ in the initial conditions (10.1). We use 1000 grid cells as before. Results are displayed in Figure 12, and should be compared with the case of no drag in Figure 3. As we can see, the drag forces here prevent the separation of two internal waves carrying a variation of $h$ and $\varphi$. Instead, after the separation of two waves carrying only a variation of $h$, we observe that a hump rich in fluid remains where the initial perturbation was located. This hump spreads laterally and its amplitude decays as time evolves. As $t \rightarrow \infty$ the flow approaches in this region the steady conditions $h=$ const. and $\varphi=$ const.

Now, we perform the computation by imposing numerically instantaneous phase velocity relaxation, and we also compute the solution of the reduced model with the same initial profiles for $h$ and $\varphi$ in (10.1), and initial velocity $v=0$. Since the relaxation scheme for the reduced model is more diffusive than the Roe-type scheme for the full two-phase model, and we wish to obtain comparable sharpness of the wave fronts, a double grid resolution (2000 grid cells) is employed for the reduced model.

We show the results of these computations in Figure 13. In each subplot we have used a thin solid line to indicate the results of the reduced model, and a thicker solid line for the results of the two-phase model with numerical infinite drag. These two sets of results are hardly distinguishable since they are approximately superimposed.

The solution of the reduced model consists of two waves carrying only a variation of $h$ moving in opposite directions, and a steady hump with higher content of fluid at the location of the initial hump. In this region the flow satisfies the equilibrium condition (5.3) of the reduced model. For this case of flat bottom this condition expresses the invariance of the hydrostatic pressure, $\mathcal{P}=\frac{g}{2} \rho h^{2}=$ const., and we see in Figure 14 that this is verified in the region of the hump. The solution of the two-phase model with infinite drag qualitatively agrees with the one of the reduced model. We notice a small decay in time of the amplitude of $h$ and $\varphi$ with respect to the solution of the reduced model that is due to the numerical errors introduced by the fractional step method (this decay for instance can be reduced by decreasing the CFL number).

\subsubsection{Dam-break problem with drag}

We now repeat the dam-break problem of Section 10.2.2 including drag effects. Results obtained by employing the drag relation in (10.11) are displayed in Figure 15. In the same figure we also plot the solution of the two-phase model without drag contribution (dotted line), which we already described, and the exact solution 

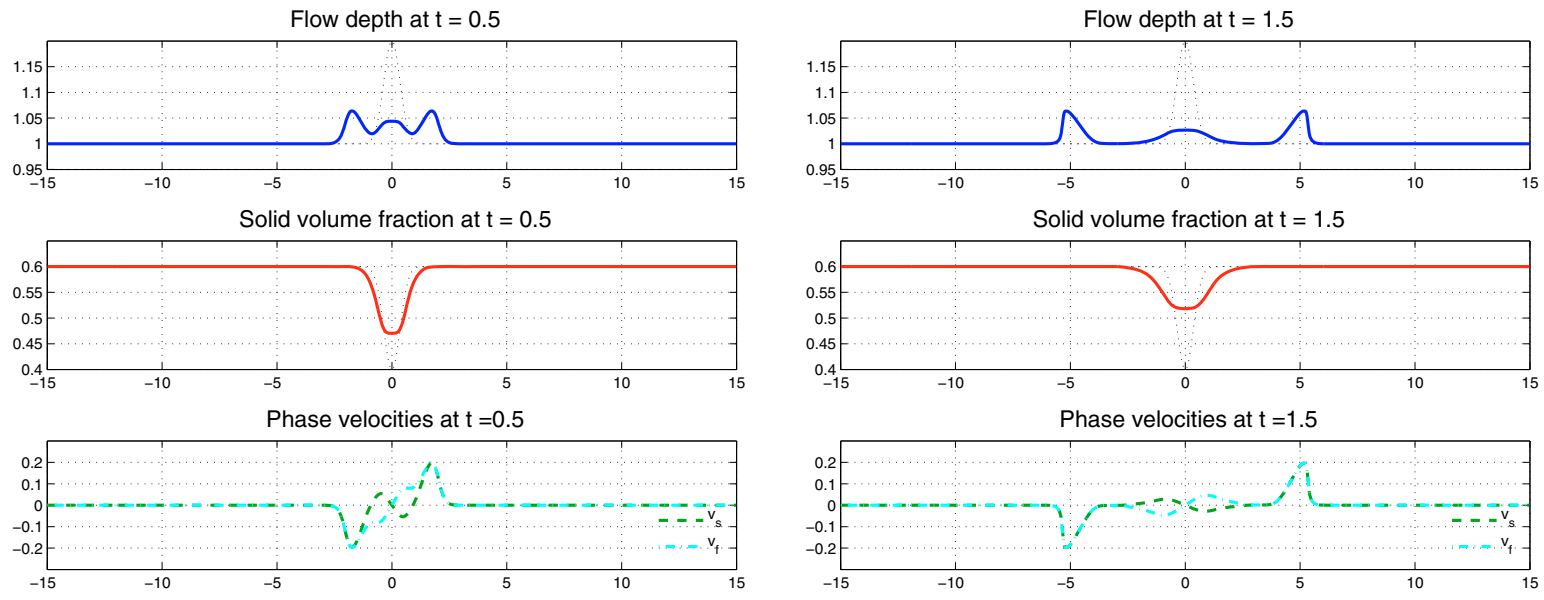

(a) $t=0.5$
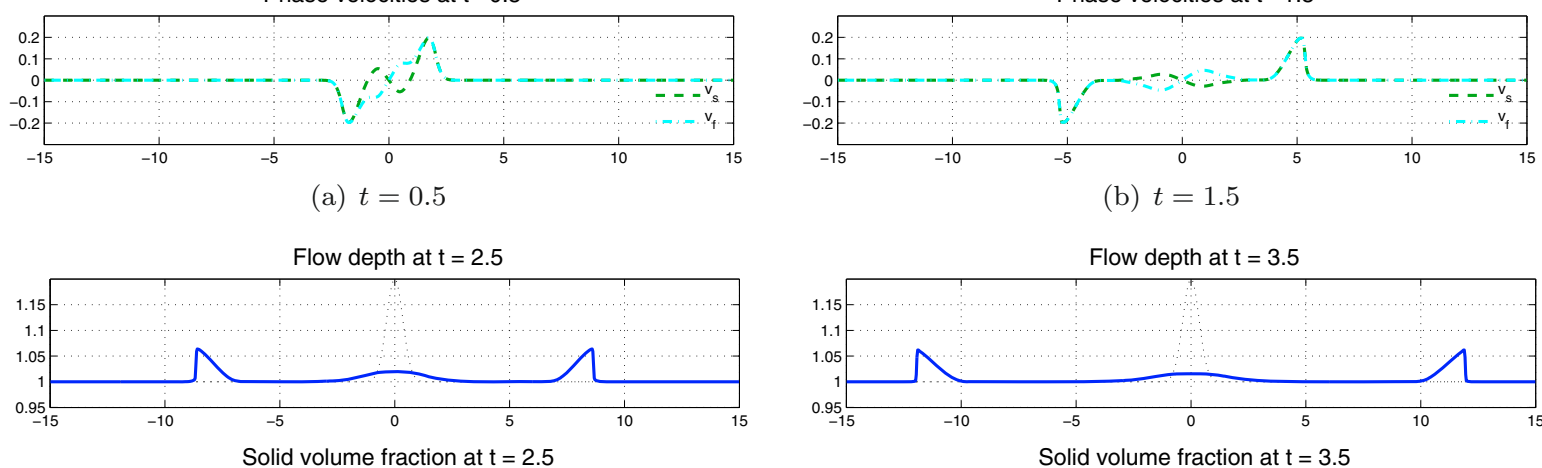

(b) $t=1.5$
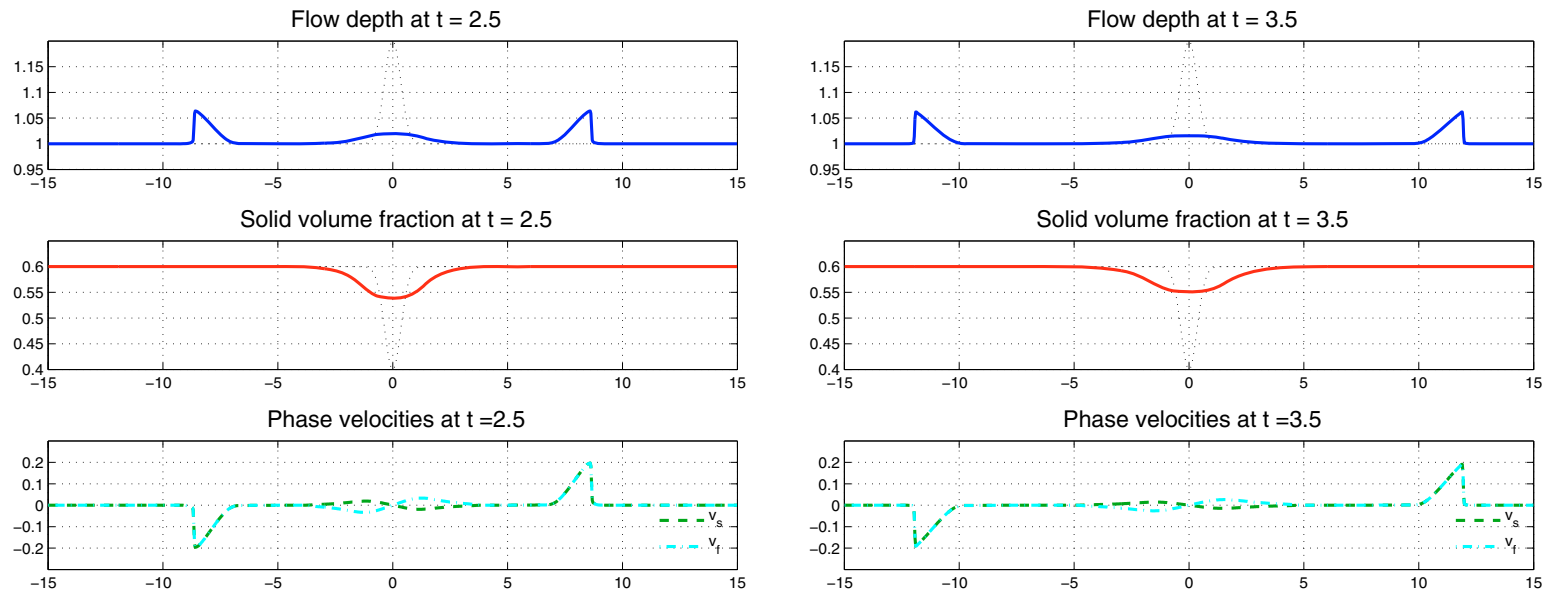

(c) $t=2.5$

(d) $t=3.5$

Figure 12. Evolution of a flow hump rich in fluid with modeling of interphase drag forces.

of the reduced model with the same initial data (dashed line). Results of the two-phase model with drag show that the reduction of the phase velocity difference in the middle region induced by drag forces causes a continuous variation of the flow height and of the solid volume fraction where there was a constant state between the 2 -shock and the 3 -rarefaction for the case with no drag.

The solution of the reduced model consists of a 1-rarefaction, a contact discontinuity and a 3-shock. As expected from the eigenstructure analysis, the solid volume fraction varies only across the contact wave, whereas the flow velocity is constant across this wave.

Finally, we compute the solution of the two-phase model in the limit of instantaneous kinematic equilibrium. The results, illustrated in Figure 16, agree with the solution of the reduced model.

\section{Concluding REMARKS}

We have introduced a new numerical model for grain-fluid mixtures over variable topography, which includes interphase drag effects. The mathematical model presents an alternative description of the fluid phase dynamics with respect to the original two-phase model of Pitman and Le. Our variant gives a conservative equation for 

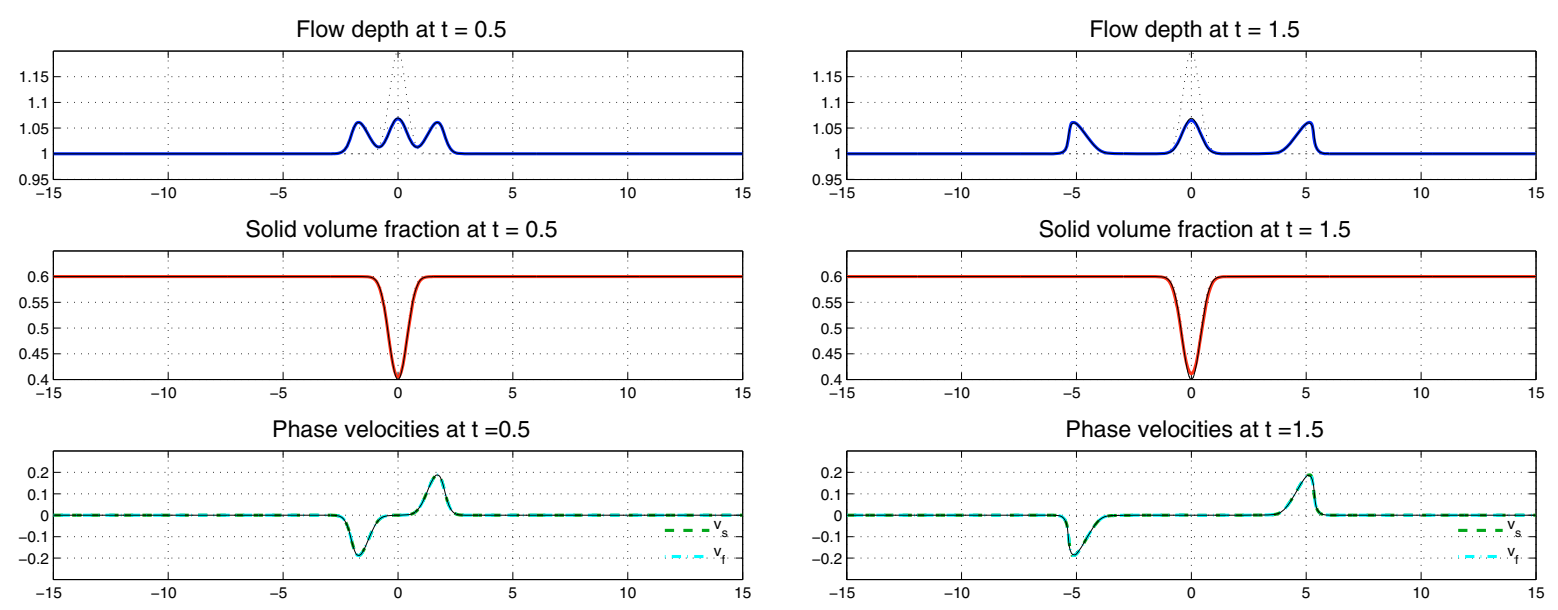

(a) $t=0.5$

(b) $t=1.5$
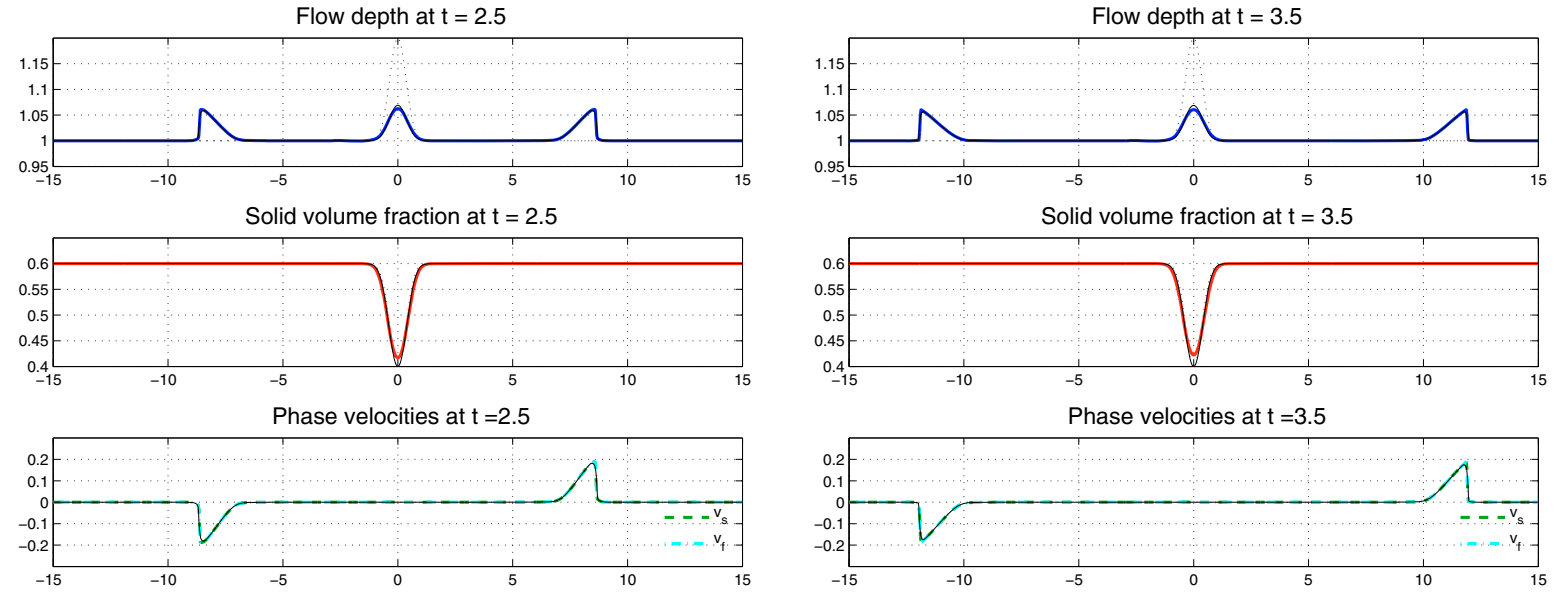

(c) $t=2.5$

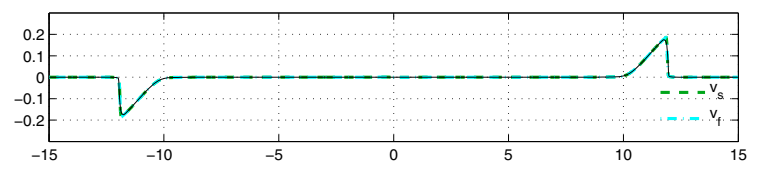

(d) $t=3.5$

FigURE 13. Evolution of a flow hump rich in fluid in the limit of instantaneous velocity equilibrium.

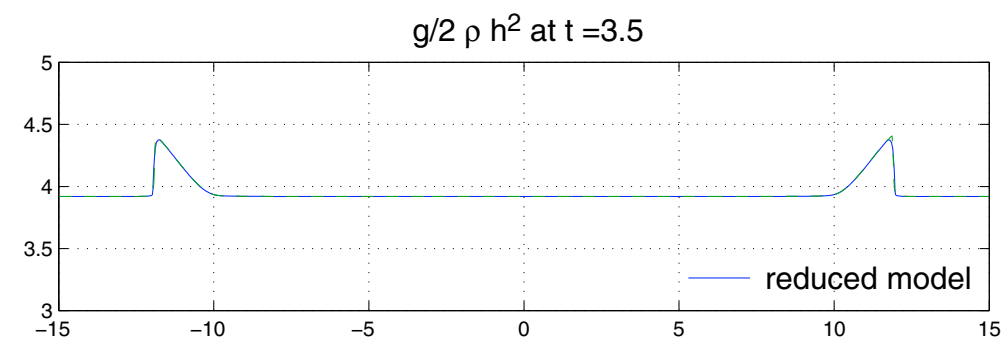

Figure 14. Profile of the hydrostatic pressure $\mathcal{P}=\frac{g}{2} \rho h^{2}$ at time $t=3.5$ for the flow hump test of Section 10.4.1. Solid line: reduced model; dashed line: two-phase model with instantaneous velocity relaxation (lines are superimposed). 

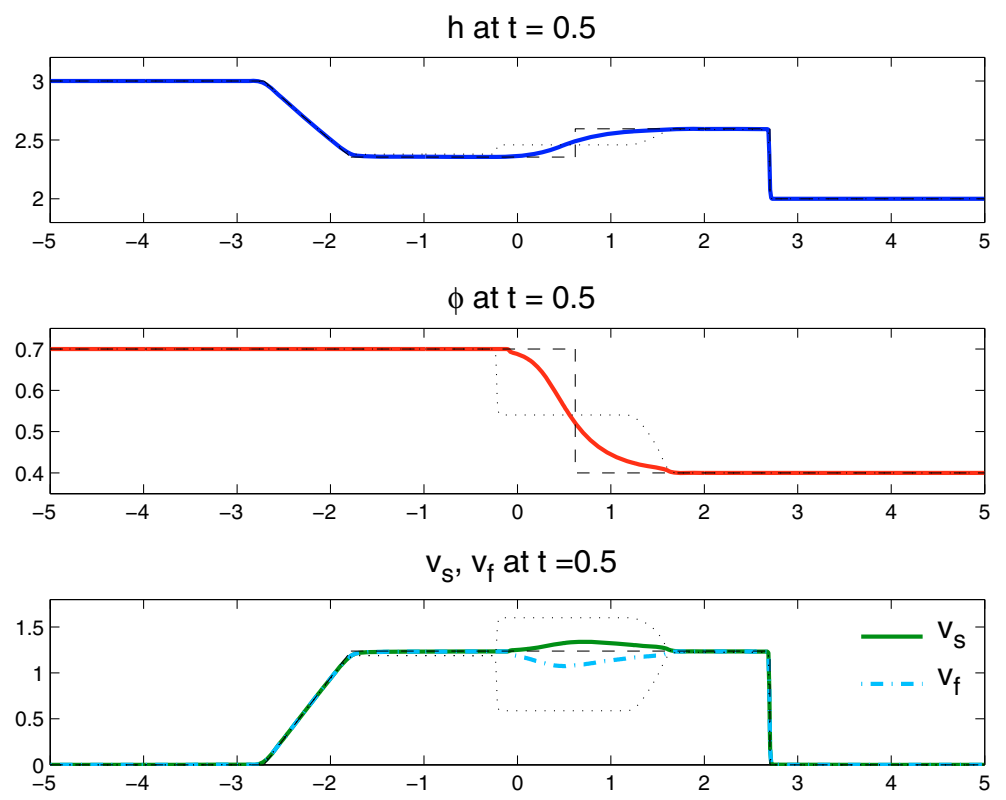

Figure 15. Dam-break problem with drag. Flow depth $h$, solid volume fraction $\varphi$, and phase velocities $v_{s}, v_{f}$ at time $t=0.5$ computed with 1000 grid cells. Dotted line: solution of the two-phase model with no drag. Dashed line: exact solution of the reduced model.
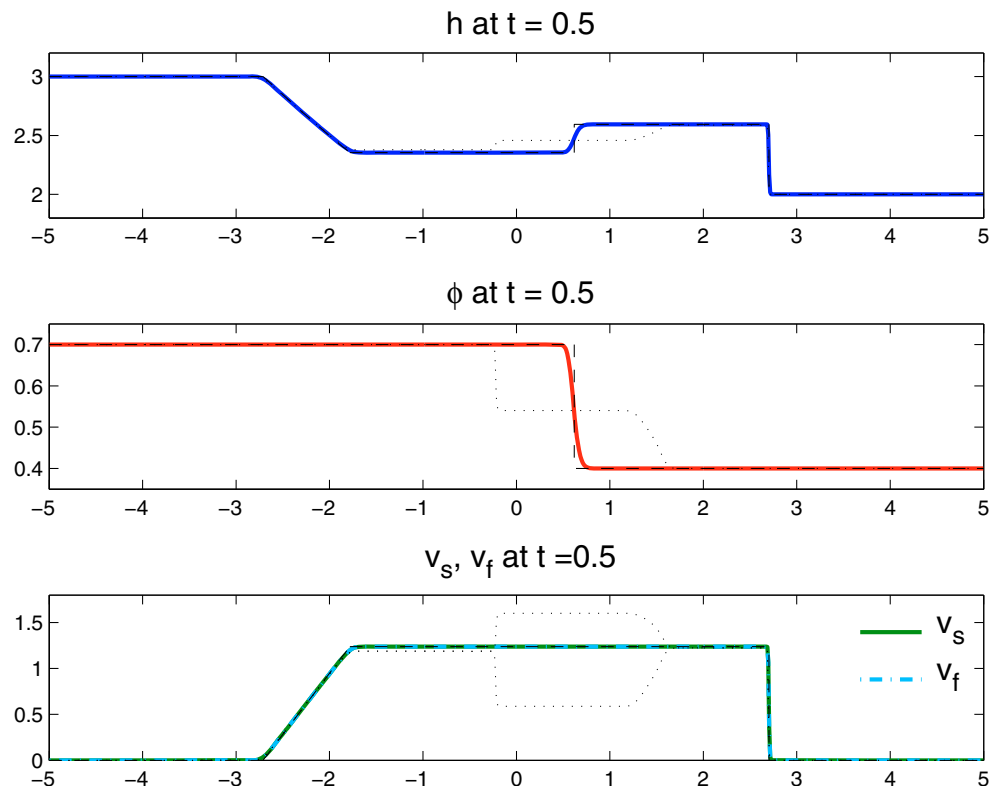

FIGURE 16. Dam-break problem in the limit of instantaneous velocity equilibrium. Flow depth $h$, solid volume fraction $\varphi$, and phase velocities $v_{s}, v_{f}$ at time $t=0.5$ computed with 1000 grid cells. Dotted line: solution of the two-phase model with no drag. Dashed line: exact solution of the reduced model. 
the momentum of the mixture, consistently with the two-phase model before averaging. Although simple explicit expressions of the system's eigenvalues cannot be derived, we were able to find precise bounding intervals for them, and to formulate sufficient conditions for the hyperbolicity of the system.

The numerical method developed for the system's solution is a finite volume scheme that uses a Roe-type Riemann solver. Topography source terms are incorporated into the Riemann solution, while drag terms are handled through a fractional-step technique. Numerical experiments demonstrate the good accuracy of the method and its well-balance property in problems of perturbed steady flows over non-flat bottom surface. Moreover, we have observed agreement of numerical results of the two-phase model in the limit of infinitely large drag with the exact solution of the reduced model that is derived theoretically by assuming that drag is strong enough to drive instantaneously phase velocities to equilibrium.

The work reported in this paper is a very first stage of a project whose ultimate goal is the development of a model applicable to realistic geophysical flows in a natural environment. One important issue that needs to be addressed in our current numerical method is guaranteeing positivity of the computed flow depth $(h \geq 0)$. Positivity preservation is an essential property for the treatment of interfaces between flow fronts and dry bed regions, that is regions where the physical flow height vanishes. Planned work includes extensions of the mathematical and numerical model to two dimensions and to flows over general bottom topography.

\section{A. Appendix. Suliciu's solver for the REduCED MOdel}

We consider here the reduced system (5.1) without topography and friction source terms. To solve numerically this system we use a Riemann solver that is an extension of Suliciu's relaxation solver for standard shallow water equations, as described in details e.g. in [4] (cf. also original works [47,48]).

First, we introduce the following relaxation system:

$$
\begin{aligned}
& \partial_{t} h+\partial_{x}(h v)=0, \\
& \partial_{t}(\rho h)+\partial_{x}(\rho h v)=0, \\
& \partial_{t}(\rho h v)+\partial_{x}\left(\rho h v^{2}+\pi\right)=0, \\
& \partial_{t}(\rho h \pi)+\partial_{x}(\rho h \pi v)+c^{2} \partial_{x} v=0,
\end{aligned}
$$

where $\pi$ is an additional variable whose equilibrium value corresponds to the hydrostatic pressure $\mathcal{P}(\rho, h)=$ $\frac{1}{2} g \rho h^{2}$, and $c$ is a parameter to be suitably chosen. To allow for a different definition of left and right values of $c$ for each Riemann problem, we then assume that $c$ is nonconstant, and that it is advected with the flow velocity. Hence, we augment the system above with the equation

$$
\partial_{t} c+v \partial_{x} c=0 .
$$

Let us consider a Riemann problem for system (A.1) with left and right data $\left(h_{\ell}, \rho_{\ell}, v_{\ell}, \pi_{\ell}, c_{\ell}\right)$ and $\left(h_{r}, \rho_{r}, v_{r}, \pi_{r}, c_{r}\right)$, respectively. Note that initially $\pi$ is set equal to its equilibrium value: $\pi_{\ell}=\mathcal{P}\left(\rho_{\ell}, h_{\ell}\right) \equiv \mathcal{P}_{\ell}$ and $\pi_{r}=\mathcal{P}\left(\rho_{r}, h_{r}\right) \equiv$ $\mathcal{P}_{r}$. The exact solution of the Riemann problem can be easily found, by observing that (A.1) can be put in the diagonal form

$$
\begin{aligned}
& \partial_{t}(\pi-c v)+(v-c / \rho h) \partial_{x}(\pi-c v)=0, \\
& \partial_{t}(\pi+c v)+(v+c / \rho h) \partial_{x}(\pi+c v)=0, \\
& \partial_{t}\left(1 / \rho h+\pi / c^{2}\right)+v \partial_{x}\left(1 / \rho h+\pi / c^{2}\right)=0, \\
& \partial_{t} \rho+v \partial_{x} \rho=0 \\
& \partial_{t} c+v \partial_{x} c=0
\end{aligned}
$$


The solution of this system consists of three linearly degenerate waves separating two intermediate states, which we will denote with indexes $\ell, *$, and $r, *$. Across the second wave $v$ and $\pi$ are invariant, thus

$$
v_{\ell}^{*}=v_{r}^{*} \equiv v^{*} \quad \text { and } \quad \pi_{\ell}^{*}=\pi_{r}^{*} \equiv \pi^{*}
$$

Moreover:

$$
\begin{array}{cl}
(\pi+c v)_{\ell}^{*}=(\pi+c v)_{\ell}, & (\pi-c v)_{r}^{*}=(\pi-c v)_{r}, \\
\left(1 / \rho+\pi / c^{2}\right)_{\ell}^{*}=\left(1 / \rho+\pi / c^{2}\right)_{\ell}, & \left(1 / \rho+\pi / c^{2}\right)_{r}^{*}=\left(1 / \rho+\pi / c^{2}\right)_{r}, \\
\rho_{\ell}^{*}=\rho_{\ell}, \quad \rho_{r}^{*}=\rho_{r}, \quad c_{\ell}^{*}=c_{\ell}, \quad c_{r}^{*}=c_{r} .
\end{array}
$$

Based on the relations above, we immediately obtain the structure of the Riemann solution. The wave speeds are

$$
v_{\ell}-\frac{c_{\ell}}{\rho_{\ell} h_{\ell}}, \quad v^{*}, \quad v_{r}+\frac{c_{r}}{\rho_{r} h_{r}},
$$

and the quantities characterizing the intermediate states are found as

$$
\begin{gathered}
v^{*}=\frac{\pi_{\ell}-\pi_{r}+c_{\ell} v_{\ell}+c_{r} v_{r}}{c_{\ell}+c_{r}}, \quad \pi^{*}=\frac{c_{r} \pi_{\ell}+c_{\ell} \pi_{r}-c_{\ell} c_{r}\left(v_{r}-v_{\ell}\right)}{c_{\ell}+c_{r}} \\
\frac{1}{h_{\ell}^{*}}=\frac{1}{h_{\ell}}+\frac{\rho_{\ell}}{c_{\ell}} \frac{\pi_{\ell}-\pi_{r}+c_{r}\left(v_{r}-v_{\ell}\right)}{c_{\ell}+c_{r}} \quad \text { and } \frac{1}{h_{r}^{*}}=\frac{1}{h_{r}}+\frac{\rho_{r}}{c_{r}} \frac{\pi_{r}-\pi_{\ell}+c_{\ell}\left(v_{r}-v_{\ell}\right)}{c_{\ell}+c_{r}} .
\end{gathered}
$$

The definition of the left and right values of $c$ is subject to the subcharacteristic condition, which requires that the characteristic speeds of the relaxation system are at least as large as the characteristic speeds of the original system (5.1). Hence we need:

$$
\begin{array}{ll}
\forall h \in\left[h_{\ell}, h_{\ell}^{*}\right], \quad \rho_{\ell} h \sqrt{g h} \leq c_{\ell}, \\
\forall h \in\left[h_{r}, h_{r}^{*}\right], \quad \rho_{r} h \sqrt{g h} \leq c_{r} .
\end{array}
$$

Furthermore, we wish to define $c_{\ell}$ and $c_{r}$ in such a way that the scheme is able to handle vacuum states. From the expression of the speeds in (A.4), we notice that we need to guarantee that the quantities $c_{\ell} /\left(\rho_{\ell} h_{\ell}\right)$ and $c_{r} /\left(\rho_{r} h_{r}\right)$ both remain bounded if either $h_{\ell}$ or $h_{r}$ goes to zero (note that at least one of them is always positive). Here, following [4], we propose to define the relaxation speeds by:

$$
\begin{aligned}
& \text { if } \mathcal{P}_{r}-\mathcal{P}_{\ell} \geq 0 \quad\left\{\begin{array}{l}
\frac{c_{\ell}}{\rho_{\ell} h_{\ell}}=\sqrt{g h_{\ell}}+\alpha\left(\frac{\mathcal{P}_{r}-\mathcal{P}_{\ell}}{\rho_{r} h_{r} \sqrt{g h_{r}}}+v_{\ell}-v_{r}\right)_{+} \\
\frac{c_{r}}{\rho_{r} h_{r}}=\sqrt{g h_{r}}+\alpha\left(\frac{\mathcal{P}_{\ell}-\mathcal{P}_{r}}{c_{\ell}}+v_{\ell}-v_{r}\right)_{+},
\end{array}\right. \\
& \text {if } \mathcal{P}_{r}-\mathcal{P}_{\ell} \leq 0 \quad\left\{\begin{array}{l}
\frac{c_{r}}{\rho_{r} h_{r}}=\sqrt{g h_{r}}+\alpha\left(\frac{\mathcal{P}_{\ell}-\mathcal{P}_{r}}{\rho_{\ell} h_{\ell} \sqrt{g h_{\ell}}}+v_{\ell}-v_{r}\right)_{+}, \\
\frac{c_{\ell}}{\rho_{\ell} h_{\ell}}=\sqrt{g h_{\ell}}+\alpha\left(\frac{\mathcal{P}_{r}-\mathcal{P}_{\ell}}{c_{r}}+v_{\ell}-v_{r}\right)_{+},
\end{array}\right.
\end{aligned}
$$

with $\alpha=3 / 2$. The only exceptions to the relations above are: (i) $c_{r} /\left(\rho_{r} h_{r}\right)=\sqrt{g h_{r}}$ if $h_{\ell}=0$, and (ii) $c_{\ell} /\left(\rho_{\ell} h_{\ell}\right)=$ $\sqrt{g h_{\ell}}$ if $h_{r}=0$. The choice of the relaxation parameters described here satisfies the conditions (A.7), and it ensures positivity of the computed values of the flow height $h$. 
Acknowledgements. The research work of the first author has been supported by a grant of the City of Paris and a grant of the Region Île-de-France. This work was also supported by the ACI Nouvelles Interfaces de Mathématiques (CNRS), by the ACI Jeunes Chercheurs (CNRS), and it is part of a project funded by the French National Research Agency (ANR Blanche, BLAN-06-1_140039).

\section{REFERENCES}

[1] T.B. Anderson and R. Jackson, A fluid-dynamical description of fluidized beds: Equations of motion. Ind. Eng. Chem. Fundam. 6 (1967) 527-539.

[2] E. Audusse, F. Bouchut, M.-O. Bristeau, R. Klein and B. Perthame, A fast and stable well-balanced scheme with hydrostatic reconstruction for shallow water flows. SIAM J. Sci. Comput. 25 (2004) 2050-2065.

[3] D. Bale, R.J. LeVeque, S. Mitran and J.A. Rossmanith, A wave-propagation method for conservation laws and balance laws with spatially varying flux functions. SIAM J. Sci. Comput. 24 (2002) 955-978.

[4] F. Bouchut, Nonlinear stability of finite volume methods for hyperbolic conservation laws, and well-balanced schemes for sources. Birkhäuser-Verlag (2004).

[5] F. Bouchut and M. Westdickenberg, Gravity driven shallow water models for arbitrary topography. Comm. Math. Sci. 2 (2004) 359-389.

[6] M.J. Castro, J. Macías and C. Parés, A Q-scheme for a class of systems of coupled conservation laws with source term. Application to a two-layer 1-D shallow water system. ESAIM: M2AN 35 (2001) 107-127.

[7] M.J. Castro, J.A. García Rodríguez, J.M. González-Vida, J. Macías, C. Parés and M.E. Vázquez-Cendón, Numerical simulation of two layer shallow water flows through channels with irregular geometry. J. Comput. Phys. 195 (2004) 202-235.

[8] R.P. Denlinger and R.M. Iverson, Flow of variably fluidized granular masses across three-dimensional terrain: 2. Numerical predictions and experimental tests. J. Geophys. Res. 106 (2001) 553-566.

[9] R.P. Denlinger and R.M. Iverson, Granular avalanches across irregular three-dimensional terrain: 1. Theory and computation. J. Geophys. Res. 109 (2004) F01014, doi:10.1029/2003JF000085.

[10] T. Gallouët, J.-M Hérard and N. Seguin, Some approximate Godunov schemes to compute shallow-water equations with topography. Comput. Fluids 32 (2003) 479-513.

[11] D. Gidaspow, Multiphase Flow and Fluidization: Continuum and Kinetic Theory Descriptions. Academic Press, New York (1994).

[12] E. Godlewski and P.-A. Raviart, Numerical Approximation of Hyperbolic Systems of Conservation Laws. Springer-Verlag, New York (1996).

[13] L. Gosse, A well-balanced flux-vector splitting scheme designed for hyperbolic systems of conservation laws with source terms. Comput. Math. Appl. 39 (2000) 135-159.

[14] N. Goutal and F. Maurel, Proceedings of the 2nd Workshop on Dam-Break Wave Simulation. Technical report EDF-DER Report HE-43/97/016/B, Chatou, France (1997).

[15] J.M.N.T. Gray, M. Wieland and K. Hutter, Gravity driven free surface flow of granular avalanches over complex basal topography. Proc. R. Soc. London S. A 455 (1999) 1841-1874.

[16] J.M. Greenberg and A.Y. LeRoux, A well-balanced scheme for the numerical processing of source terms in hyperbolic equations. SIAM J. Numer. Anal. 33 (1996) 1-16.

[17] A. Harten and J.M. Hyman, Self-adjusting grid methods for one-dimensional hyperbolic conservation laws. J. Comput. Phys. 50 (1983) 235-269.

[18] K. Hutter, M. Siegel, S.B. Savage and Y. Nohguchi, Two-dimensional spreading of a granular avalanche down an inclined plane, part I. Theory. Acta Mech. 100 (1993) 37-68.

[19] R.M. Iverson, The physics of debris flows. Rev. Geophys. 35 (1997) 245-296.

[20] R.M. Iverson and R.P. Denlinger, Flow of variably fluidized granular masses across three-dimensional terrain: 1, Coulomb mixture theory. J. Geophys. Res. 106 (2001) 537-552.

[21] R.M. Iverson, M. Logan and R.P. Denlinger, Granular avalanches across irregular three-dimensional terrain: 2, Experimental tests. J. Geophys. Res. 109 (2004) F01015, doi:10.1029/2003JF000084.

[22] F. Legros, The mobility of long-runout landslides. Eng. Geol. 63 (2002) 301-331.

[23] R.J. LeVeque, CLAWPACK. http://www.amath.washington.edu/ claw.

[24] R.J. LeVeque, Wave propagation algorithms for multi-dimensional hyperbolic systems. J. Comput. Phys. 131 (1997) $327-353$.

[25] R.J. LeVeque, Balancing source terms and flux gradients in high-resolution Godunov methods: The quasi-steady wavepropagation algorithm. J. Comput. Phys. 146 (1998) 346-365.

[26] R.J. LeVeque, Finite Volume Methods for Hyperbolic Problems. Cambridge University Press (2002).

[27] R.J. LeVeque and D.L. George, High-resolution finite volume methods for the shallow water equations with bathymetry and dry states, in Proceedings of Long-Wave Workshop, Catalina, 2004, P.L.-F. Liu, H. Yeh and C. Synolakis Eds., Advances Numerical Models for Simulating Tsunami Waves and Runup, Advances in Coastal and Ocean Engineering 10, World Scientific (2008) 43-73. 
[28] R.J. LeVeque and M. Pelanti, A class of approximate Riemann solvers and their relation to relaxation schemes. J. Comput. Phys. 172 (2001) 572-591.

[29] A. Mangeney, F. Bouchut, N. Thomas, J.-P. Vilotte and M.-O. Bristeau, Numerical modeling of self-channeling granular flows and of their levee-channel deposits. J. Geophys. Res. 112 (2007) F02017, doi:10.1029/2006JF000469.

[30] A. Mangeney-Castelnau, J.-P. Vilotte, M.-O. Bristeau, B. Perthame, F. Bouchut, C. Simeoni and S. Yernini, Numerical modeling of avalanches based on Saint-Venant equations using a kinetic scheme. J. Geophys. Res. 108 (2003) 2527, doi:10.1029/2002JB002024.

[31] A. Mangeney-Castelnau, F. Bouchut, J.-P. Vilotte, E. Lajeunesse, A. Aubertin and M. Pirulli, On the use of Saint-Venant equations to simulate the spreading of a granular mass. J. Geophys. Res. 110 (2005) B09103, doi:10.1029/2004JB003161.

[32] M. Massoudi, Constitutive relations for the interaction force in multicomponent particulate flows. Int. J. Non-Linear Mech. 38 (2003) 313-336.

[33] S. Noelle, N. Pankratz, G. Puppo and J.R. Natvig, Well-balanced finite volume schemes of arbitrary order of accuracy for shallow water flows. J. Comput. Phys. 213 (2006) 474-499.

[34] C. Parés and M.J. Castro, On the well-balance property of Roe's method for nonconservative hyperbolic systems. Applications to shallow-water systems. ESAIM: M2AN 38 (2004) 821-852.

[35] A.K. Patra, A.C. Bauer, C.C. Nichita, E.B. Pitman, M.F. Sheridan, M. Bursik, B. Rupp, A. Webber, A.J. Stinton, L.M. Namikawa and C.S. Renschler, Parallel adaptive numerical simulation of dry avalanches over natural terrain. J. Volcanology Geotherm. Res. 139 (2005) 1-21.

[36] M. Pelanti, Wave Propagation Algorithms for Multicomponent Compressible Flows with Applications to Volcanic Jets. Ph.D. thesis, University of Washington, USA (2005).

[37] M. Pelanti and R.J. LeVeque, High-resolution finite volume methods for dusty gas jets and plumes. SIAM J. Sci. Comput. 28 (2006) 1335-1360.

[38] E.B. Pitman and L. Le, A two-fluid model for avalanche and debris flows. Phil. Trans. R. Soc. A 363 (2005) $1573-1601$.

[39] E.B. Pitman, C.C. Nichita, A.K. Patra, A.C. Bauer, M.F. Sheridan and M. Bursik, Computing granular avalanches and landslides. Phys. Fluids 15 (2003) 3638-3646.

[40] S.P. Pudasaini and K. Hutter, Rapid shear flows of dry granular masses down curved and twisted channels. J. Fluid Mech. 495 (2003) 193-208.

[41] S.P. Pudasaini, Y. Wang and K. Hutter, Modelling debris flows down general channels. Natural Hazards and Earth System Sciences 5 (2005) 799-819.

[42] S.P. Pudasaini, Y. Wang and K. Hutter, Rapid motions of free-surface avalanches down curved and twisted channels and their numerical simulations. Phil. Trans. R. Soc. A 363 (2005) 1551-1571.

[43] W.J.M. Rankine, On the stability of loose earth. Phil. Trans. R. Soc. 147 (1857) 9-27.

[44] P.L. Roe, Approximate Riemann solvers, parameter vectors, and difference schemes. J. Comput. Phys. 43 (1981) 357-372.

[45] S.B. Savage and K. Hutter, The motion of a finite mass of granular material down a rough incline. J. Fluid. Mech. 199 (1989) $177-215$.

[46] S.B. Savage and K. Hutter, The dynamics of avalanches of granular materials from initiation to runout, part I. Analysis. Acta Mech. 86 (1991) 201-223.

[47] I. Suliciu, On modelling phase transitions by means of rate-type constitutive equations, shock wave structure. Internat. J. Engrg. Sci. 28 (1990) 829-841.

[48] I. Suliciu, Some stability-instability problems in phase transitions modelled by piecewise linear elastic or viscoelastic constitutive equations. Internat. J. Engrg. Sci. 30 (1992) 483-494.

[49] Y.C. Tai, S. Noelle, J.M.N.T. Gray and K. Hutter, Shock-capturing and front-tacking methods for dry granular avalanches. J. Comput. Phys. 175 (2002) 269-301.

[50] E.F. Toro, Riemann Solvers and Numerical Methods for Fluid Dynamics. Springer-Verlag, Berlin, Heidelberg (1997).

[51] B.G.M. van Wachem and A.E. Almstedt, Methods for multiphase computational fluid dynamics. Chem. Eng. J. 96 (2003) $81-98$.

[52] M.E. Vázquez-Cendón, Improved treatment of source terms in upwind schemes for the shallow water equations in channels with irregular geometry. J. Comput. Phys. 148 (1999) 497-526.

[53] P. Vollmöller, A shock-capturing wave-propagation method for dry and saturated granular flows. J. Comput. Phys. 199 (2004) $150-174$.

[54] C.B. Vreugdenhil, Two-layer shallow-water flow in two dimensions, a numerical study. J. Comput. Phys. 33 (1979) $169-184$.

[55] Y. Wang and K. Hutter, A constitutive model of multiphase mixtures and its application in shearing flows of saturated solid-fluid mixtures. Granul. Matter 1 (1999) 163-181.

[56] Y. Wang and K. Hutter, A constitutive theory of fluid-saturated granular materials and its application in gravitational flows. Rheol. Acta 38 (1999) 214-223. 